\title{
斯金纳自动机：形成操作性条件反射理论的 心理学模型
}

\author{
阮晓钢，武璇* \\ 北京工业大学电子信息与控制工程学院人工智能与机器人研究所, 北京 100124 \\ *E-mail: abc0767@live.com
}

收稿日期: 2013-08-06; 接受日期: 2013-09-13

国家重点基础研究发展计划(“973”计划)(批准号: 2012CB720000)、国家高技术研究发展计划(“863”计划)(批准号: 2007AA04Z226)、国家 自然科学基金(批准号: 61075110, 60774077, 61375086)、北京市自然科学基金(批准号: 4102011)、北京市教育委员会科技发展计划重点(批 准号: KM200810005016, KZ201210005001)和教育部高等学校博士学科点专项科研基金(批准号: 20101103110007)资助项目

\begin{abstract}
摘要操作性条件反射是动物学习的一种基本的机制, 它表示所有的动物, 从微生物到 人类都是通过操作性条件反射来指导学习的. 介绍了一种新的概率自动机, 称作斯金纳自 动机, 它是形成操作性条件反射的一种心理学模型. 通过热力学过程来模仿动物的学习过 程, 并且从蒙特卡罗方法以及 Metropolis 算法, 和模拟退火算法中提出了斯金纳算法. 在 特定条件下, 证明了斯金纳自动机是便利的、 $\varepsilon$-最优的、最优的以及操作概率会以概率 1 收缩到一个稳定的根. 斯金纳自动机使机器可以像动物一样自主地学习.
\end{abstract}

关键词

学习自动机

波尔兹曼分布

操作性条件反射

操作性学习

模拟退火算法

\section{1 引言}

操作性条件反射是行为心理学的基本概念之一, 因为斯金纳的贡献, 有时也称为斯金纳条件反射 ${ }^{[1,2]}$. 早期的工作可以追溯到美国心理学家 Thorndike ${ }^{[3]}$ 的 研究, 他研究了在猫, 狗, 鸡, 猴中的反复试验, 不 断摸索的学习方式, 提出了著名的效果律, 成为了斯 金纳工作的概念的开端. 行为主义的奠基人 Watson ${ }^{[4]}$ 证明了行为的研究可以是系统地, 而不依赖于内在 心理状态 ${ }^{[5]}$. 斯金纳是一个激进的行为主义者, Watson 对他产生的影响很大. 然而, 斯金纳认为在 Watosn 的理论中存在着一系列的缺点, 它们都建立 在 Pavlov 条件反射之上 ${ }^{[6]}$. 在斯金纳看来, Pavlov 条 件反射过程中, 生物都过于被动和无意识. 他相信生 物都是主动地并且有意识地与环境互动 ${ }^{[1]}$. 斯金纳把
主动的行为称为自发反应 ${ }^{[2]}$, 由此操作性条件反射得 以命名.

操作性条件反射是心理学学习的一种重要的形 式, 有时被称为操作性学习, 在这个过程中人类和动 物学会了将他们的行为与产生的结果联系起来. 操 作性学习启发了机器学习方面的研究. 有一些数学 模型也在试图模拟操作性条件反射. 早期的工作有 Grossberg $^{[7,8]}$ 提出的用理想化的神经网络来为操作性 条件反射建模, 用来解释脊椎动物学习时的行为. Grossberg 的模型随后被 Chang 和 Gaudiano ${ }^{[9]}$ 用在机 器人上来学习壁障的自发行为. Touretzky 和他的团 队 ${ }^{[10 ~ 12]}$ 研究了使机器人 Skinnerbot 可以通过操作性 条件反射模型训练的课题. Itoh 等人 ${ }^{[13,14]}$ 认为机器人 需要像人一样表达情感, 行为和个性. 他们提出了一 个操作性条件反射的行为模型并且应用在了一个类 
人的情感表达机器人(WE-4RII)上, 使机器可以自发 地选择适当的行为.

强化学习是机器学习的一种重要形式, 它是受 行为主义心理学启发而来. 操作性条件反射是强化 学习的基础. 事实上, 强化的概念就是从操作性条件 反射的理论中得出的, 它在操作性条件反射中扮演 了重要的角色. Sutton 和 Barto ${ }^{[15]}$ 奠定了强化学习的 基本原理强化学习可以追溯到学习自动机的研究上. 术语“学习自动机”最早被 Narendra 和 Thathachar ${ }^{[16]}$ 提出, 他们指出最早的学习自动机是在数学心理学 中发展来的. 原始的学习自动机概念是所谓的有限 动作集学习自动机. 有限动作集学习自动机有一套 给定的动作集合以及特定的强化策略, 它在一个未 知的随机环境中活动, 并且根据环境反应来更新动 作集中动作的概率. 明显地, 学习自动机也是受行为 心理学启发而来. 学习自动机是操作性学习的模型, 也是强化学习形式化的框架.

学习自动机的观念多年来一直在拓展 ${ }^{[17]}$. 有限 动作集学习自动机确保了只能收玫到强化信号的局 部极大上 ${ }^{[18,19]}$. 为了收玫到全局最大, 参数化学习自

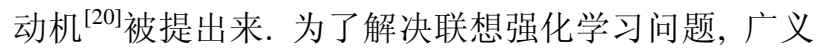
学习自动机被提出 ${ }^{[21,22]}$. 它们之间的共同之处就是 强化策略作为学习过程的基础 ${ }^{[2327]}$. 根据线性特性, 强化策略可以分为线性, 非线性以及混合策略. 学习 自动机中的强化策略是对学习自动机性能有着至关 重要的影响的. 根据用不同强化策略的学习自动机 展现出的不同特性, 可以将它们分为便利、 $\varepsilon$-最优、 最优和绝对便利.

就像 Touretzky 和 Saksida 指出的, 动物通过操作 性条件反射可以比机器通过强化学习或者学习自动 机学到复杂得多的行为. 在这篇文章中, 我们提出了 一种叫做斯金纳自动机的新的心理学模型来使操作 性条件反射理论形式化. 我们把动物的操作性学习 看作是热力学过程, 从 Metropolis 算法 ${ }^{[28,29]}$ 和模拟退 火算法 ${ }^{[30,31]}$ 导出了斯金纳算法. 斯金纳算法是斯金 纳自动机的核心, 它是斯金纳自动机的强化策略. 模 拟退火算法确保了斯金纳自动机可以收敛到全局最 优. 我们的理论分析显示斯金纳自动机不仅是便利 的以及 $\varepsilon$-最优的, 也是最优的和绝对便利的. 在文章 的后面，一些优化计算和模拟实验被用来展示斯金 纳自动机的学习效果. 我们的工作揭示了操作性条件 反射不仅是心理学的和生物学的, 也是可计算的, 心
理动力学的以及热力学的. 斯金纳自动机可以使包括 机器人在内的自主智能体自主地像动物一样学习.

文章剩下的部分组织如下：第 2 节介绍了操作性 条件反射. 第 3 节结合操作性条件反射提出了斯金纳 自动机并描述了斯金纳算法是斯金纳自动机的核心. 第 4 节讨论了斯金纳自动机的自组织特性. 第 5 和 6 节通过斯金纳自动机再现了著名的斯金纳鸽子实验. 第 7 节展示了一个虚拟的三维维纳虫, 通过斯金纳自 动机作为它的趋光动作系统, 它通过操作性条件反 射学习形成了负趋光特性. 第 8 节中, 斯金纳自动机 作为一个两轮柔性机器人的感觉运动系统, 使机器 人逐渐通过操作性条件反射学习自主地掌握了平衡 技能. 最后, 第 9 节是结语.

\section{2 操作性条件反射的相关分析}

一个对机器学习自然的问题就是它能否与动物 学习同构. 我们需要使操作性条件反射理论在类似 学习自动机的条件下形式化, 这样人工系统就可以 在操作性条件反射的方式下像动物一样学习. 在心 理动力学中 ${ }^{[32]}$, 操作性条件反射是一个动力学过程. 学习自动机是动力学系统. 在这节中, 将学习自动机 中的操作性条件反射的动力学过程还原, 使之与动 物学习中的操作性条件反射同构.

\section{1 学习自动机}

这里将简短描述学习自动机的理论, 来为理解 学习自动机和操作性条件反射的学习过程的比较提 供背景.

一般地, 学习自动机是一种在随机环境中运动 的随机动力系统, 可以通过一个七元组来定义:

$$
L A=\langle t, S, \alpha, \beta, F, G, A\rangle,
$$

其中 $t \in\{0,1,2, \cdots\}$ 表示学习自动机在学习过程中的离 散时间, $S$ 是内部状态集合, $\alpha$ 是动作集合, 也是学习 自动机的输出, $\beta$ 是环境的反应集合, 也是学习自动 机的输入集, $F: S \times \beta \rightarrow S$ 是一个将目前状态与输入映 射到下一个状态的函数, 他可以是确定性的也可以 是随机性的, $G: S \rightarrow \alpha(p)$. $G$ 是根据发生概率 $p$ 把当前 状态映射为输出 ( $\alpha$ 中的动作)的函数, $A$ 是根据当前 行为的概率, 行为以及输入来产生下一个行为的算 法(也称作更新策略或强化策略). 
学习自动机的动力学过程如图 1 所示, 学习自动 机及其环境的互动形成了一个闭环反馈连接. 动作 $\alpha(t)$ 是学习自动机 $t$ 时刻的输出, 作用在环境上并引 起反应 $\beta(t)$ 作为给学习自动机的反馈以及 $t$ 时刻的输 入使学习自动机选择下一个行为 $\alpha(t+1)$.

(1)式中的学习自动机有两个动力学特性: 状态 转移和学习更新. 在任意时刻, 学习自动机的下一个 状态不仅取决于当前的输入, 还取决于当前的状态. 学习自动机通过在环境反应的基础上更新概率来学 习. 下一个行为的概率不仅取决于当前行为和环境 的反应, 也取决于当前的行为概率.

(1)式中的学习自动机有 3 个基本元素: 1) 动作 集合 $(\alpha), 2)$ 强化刺激集合 $(\beta)$, 以及 3$)$ 强化策略 $(A)$. 看起来学习自动机可以用操作性条件反射的方式来 进行强化学习, 强化策略 $A$ 总是试图奖励好的行为并 且惩罚坏的行为. 然而用心理动力学的话说 ${ }^{[32]}$, 在学 习自动机的概率更新过程和动物的操作性条件反射 过程中, 还是存在着不小的差异.

\section{2 操作性条件反射中行为和结果的关联}

所有活的生物都是动力学系统. 在系统动力学 中 $^{[33]}$, 在任意给定的时间生物都有其内部状态, 既是 心理上的也是生理上的, 并且随着时间一直变化着.

操作性条件反射是心理动力学和生理动力学过 程的一种形式, 使行为和结果产生了联系. 在操作性 条件反射中, 行为是自发的以及主动的, 称为自发反 应. 自发性的，按斯金纳的话说 ${ }^{[2]}$ 是操作性条件反射

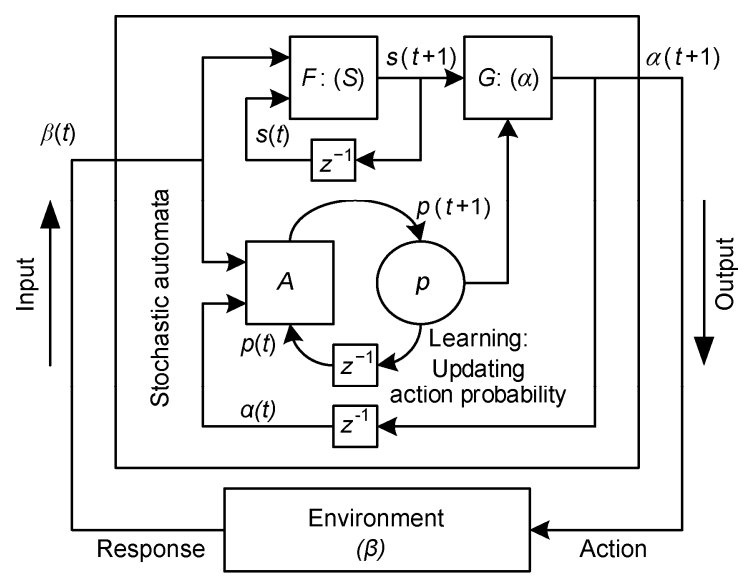

图 1 学习自动机和它的动力学过程

$\alpha(t), \beta(t)$ 和 $p(t)$ 分别代表了学习自动机的动作集合、环境的反应以及 $t$ 时刻的行为的概率
理论的一个核心概念, 它反映了行为主义的基本特 性并且有双重的重要暗示：1) 它是生物的自发性的 和主动的行为,2) 它会产生特定的结果.

在某种意义上, 操作性条件反射中操作性行为 的概念与学习自动机中动作的概念是相同的.

生物的任何操作性行为都会有一个特定的结果, 然后这个结果又引起新的操作性行为. 在操作性条 件反射的过程中, 操作性行为和结果的交替出现形 成了生物内部通过环境形成的反馈循环, 如图 2, 这 其中包括联系了操作性行为和结果的前向反馈和后 向反馈. 在操作性条件反射过程中, 结果又是被认为 是刺激, 行为(操作性行为)被认为是反应, 因此操作 性条件反射又称为反应-刺激条件反射. 在操作性条 件反射的闭环中前向反馈是一种刺激-反应过程, 它 代表了生物的行为选择, 或者在操作性条件反射中 称为操作性行为选择. 反之, 后向反馈是一种反应刺激过程, 代表了生物的状态转移.

在学习自动机理论中, 来自环境的输入被看作 是动作产生的结果, 如强化刺激, 对于动作既不是奖 励也不是惩罚. 然而, 我们认为在操作性条件反射过 程中, 来自环境的反应并不真正是操作性行为的结 果, 它只是中间的结果. 真正的由操作性行为引起的 结果是在操作性条件反射中生物状态的改变. 例如, 对一个操作性行为的奖励不是这个操作性行为的最 终结果, 只是在操作性行为和它的结果的中间物, 操 作性行为引起的真正的结果是使奖励可以给生物带 来内部状态特定的满足感. 因此, 在操作性条件反射 中, 操作性行为的结果可以认为是生物状态的转变. 在一定程度上, 生物当前的状态代表了上一个操作

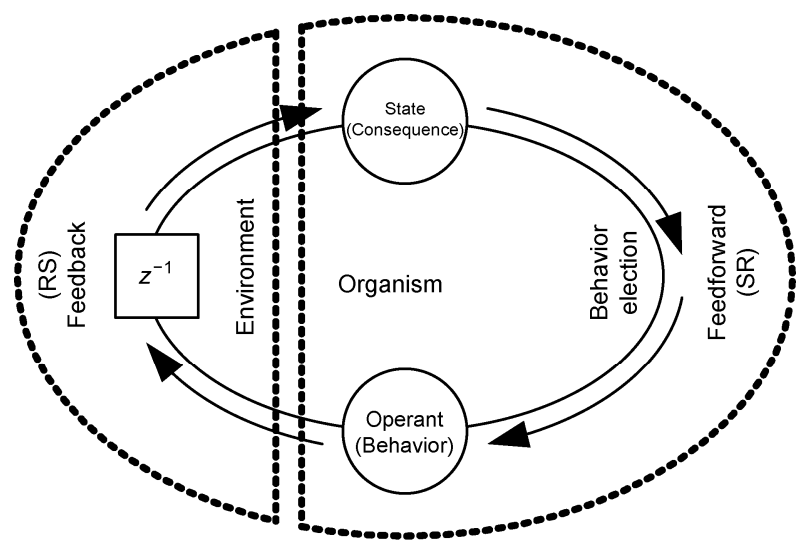

图 2 操作性条件反射中行为和结果交替出现的闭环图 
性行为的结果.

因此，在操作性条件反射中的刺激-反应前向反 馈过程中操作性行为的选择可以用学习自动机(1)中 的函数 $G(p): S \rightarrow \alpha$ 来表达. 一些学习自动机将行为选 择写成公式 $G: S \times \beta \rightarrow \alpha(p)$ 这意味着学习自动机中的 行为选择依赖于学习自动机的状态以及来自环境的 输入. 然而像上面说的, 来自环境的输入的效果可以 认为包含在操作性条件反射中的生物的状态中. 因 此, 在每个时刻 $t$, 操作性条件反射中操作性行为的 选择可以计算地推广为

$$
\mathrm{SR}: s(t) \stackrel{p(o(t) \mid s(t))}{\rightarrow} o(t),
$$

其中 $o(t)$ 和 $s(t)$ 分别是当前状态和操作性行为, 并且 $p(o(t) \mid s(t))$ 是操作性行为 $o(t)$ 在状态 $s(t)$ 下发生的概率. (2)式将操作性条件反射中的结果(状态)到操作性行 为的刺激一反应前向反馈公式化了, 这说明了操作性 条件反射中生物完全在当前状态的基础上选择它当 前的操作性行为.

(1)式中所示的学习自动机的状态转移通过函数 $F: S \times \beta \rightarrow S$ 而来, 这说明学习自动机的状态转移是来 自环境的输入推动的. 然而, 如之前证明的, 操作性 条件反射中生物的状态转移可以看作是操作性行为 引起的结果. 因此操作性条件反射的状态转移并不 真正地依赖于来自环境的反应, 而是依赖于生物自 身的操作性行为. 所以在每个时刻 $t$, 操作性条件反 射中生物的状态转移可以计算地推广为

$$
\mathrm{RS}: s(t) \times o(t) \rightarrow s(t+1),
$$

其中 $o(t)$ 和 $s(t)$ 分别是当前的状态和操作性行为, $s(t+1)$ 是下一个状态. (3)式将操作性行为到结果过程中的 反应-刺激反馈公式化, 说明操作性行为是驱动生物 状态转移的能源, 而且生物未来的状态取决于当前 状态和操作性行为.

实际上, 在操作性条件反射中, 生物的状态转移 过程是一个黑箱. 生物可以感觉到它自己的状态或 者状态的改变, 但是不能分辨其过程. 我们需要注意 (1)和(3)的不同. 操作性条件反射中的状态转移直接 由操作性行为推动, 但是在学习自动机中, 是由环境 的反应推动的.

\section{3 操作性条件反射中的动机和强化}

强化是操作性条件反射的一个关键概念 ${ }^{[34]}$, 它
被定义为通过给出一个刺激, 如加强或者奖励操作 性行为来增加一个操作性行为发生概率的事件. 反 之降低概率就是惩罚. 强化在形成或者改变人和动 物的行为中扮演了重要的角色. 如果认为强化不是 正的就是负的, 那么奖励和惩罚也可以看作是强化. 计算方面来讲, 正的强化刺激代表了奖励, 负的代表 惩罚.

在操作性条件反射过程中, 生物似乎倾向于更 多地展示可以得到奖励的行为, 更少地展现得到惩 罚的行为. 然而, 我们认为对于特定的心理和生理感 觉或者状态上的满足, 生物并不那么迫切寻求它们. 冊庸置疑, 在操作性条件反射或者自发性学习中, 生 理和心理上的感觉或者状态是最激进的强化刺激. 事实上, 在操作性条件反射过程中, 任何强化的效果 都将最终反应在生物的心理以及生理的感觉或者状 态上. 幸福、愉快以及舒适等都是正的强化刺激使操 作性行为的发生概率趋向于增加, 反之, 悲伤、失望、 沮丧、忧郁等都是负强化刺激, 会使操作性行为的发 生概率趋于减小.

在操作性条件反射中强化是与动机相关的 ${ }^{[35,36]}$, 动机使生物采取行动或者操作性行为. 动机在操作 性条件反射中是一种发起, 指导, 并且维持趋向于目 标的行为的力量. 操作性条件反射在生物的心理和 生理感觉与动机一致时或生物的状态转移时趋向于 动机的目标趋向于提高正强化, 反之亦然.

操作性条件反射的特性可以通过心理动力学 ${ }^{[32]}$ 来刻画, 它是心理学和热力学的混合. 心理动力学的 奠基人 Bruck $\mathrm{e}^{[37]}$ 提出所有的生物都是符合热力学第 一定律的能量系统. 参考生物能量学, 它也可以通过 生物热力学来刻画 ${ }^{[38,39]}$, 并且研究生物钟能量的传 导. 巧合的是, 心理动力学和生物热力学都受到热力 学的启发并与能量有关, 这就意味着在操作性条件 反射过程中, 生物的或者心理的与生理的状态需要 能量来保持, 并且操作性行为也需要能量来执行. 因 此, 在操作性条件反射中生物可以说是一个能量系 统 $\mathcal{O}_{\mathrm{SYS}}$ 由两个函数组成: 一个是内能函数 $\mathcal{O}_{\mathrm{S}}$ 给每一 个生物状态非负能量值; 另一个是操作性行为能量 函数 $\widetilde{\mathscr{O}_{0}}$ 给每个操作性行为非负能量值, 即

$$
\begin{aligned}
& \mathcal{E}_{\mathrm{SYS}}(t)=\Delta \mathcal{E}_{\mathrm{S}}(t)+\mathscr{E}_{\mathrm{O}}(t), \\
& \mathcal{E}_{\mathrm{S}}: s(t) \rightarrow R^{+}, \quad \tilde{\mathcal{E}}_{\mathrm{O}}: o(t) \rightarrow R^{+},
\end{aligned}
$$

其中 $\Delta \mathscr{\mathscr { E }}_{\mathrm{S}}(t)=\widetilde{\mathscr{G}}_{\mathrm{S}}(t+1)-\widetilde{\mathscr{E}}_{\mathrm{S}}(t)$ 是内能从 $t$ 到 $t+1$ 时刻的增量. 
内能 $\mathcal{E}_{\mathrm{S}}$ 是生物保持状态的潜在能量, 操作性行为能 量 $\mathscr{E}_{0}$ 是生物执行操作性行为的能量. (4)式说明在操 作性条件反射中, 伴随着内能的变化以及操作性行 为能量的消耗, 生物的状态转移是由操作性行为驱 动的. 在每个时刻 $t, \varepsilon_{S Y S}$ 通过一个非负的能量值估计 了操作性行为 $o(t)$ 在状态 $s(t)$ 的结果.

通常假设低能量状态与生物的趋向性是一致 的 ${ }^{[40]}$. 在操作性条件反射中生物的趋向性可以假设为 通过最少能量的操作来保持低能量状态. 因此, 在操 作性条件反射中可以通过具有建立在能量系统 $\tilde{\sigma}_{\mathrm{SYS}}$ 上 的目标函数的动机系统 $M_{\mathrm{SYS}}$ 来刻画生物的动机:

$$
M_{\mathrm{SYS}}: J(o(t))=\sum_{t=0}^{+\infty} \mathcal{E}_{\mathrm{SYS}}(t),
$$

其中目标函数 $J$ 也叫做自发性指数. (5)式说明操作性 条件反射的目标的动机计算地指示了最小化自发性 指数并且获得最优操作性行为序列 $\left\{o^{*}(t)\right\}$ 的目的. 随 后, 操作性条件反射的强化可以像更新操作性行为 的概率一样计算地形成:

$$
\mathrm{RF}: \Delta p(o(t) \mid s(t)) \begin{cases}>0, & \tilde{\mathcal{E}}_{\mathrm{SYS}}(t)<0, \\ <0, & \tilde{\mathcal{E}}_{\mathrm{SYS}}(t)>0,\end{cases}
$$

其中 $p(o(t) \mid s(t))$ 是操作性行为 $o(t)$ 在状态 $s(t)$ 下的发生 概率, $\Delta p(o(t) \mid s(t))$ 是发生概率 $p(o(t) \mid s(t))$ 的增量, $\varepsilon_{S Y S}(t)$ 是(4)中能量系统的能量值.

操作性条件反射的动机和强化如图 3 所示, 操作 性条件反射的过程可以通过概率更新的过程来说明. 在(6)的强化策略中, 操作性行为 $o(t)$ 在状态 $s(t)$ 下通 过给出发生概率 $p(o(t) \mid s(t))$ 的增量 $\Delta p(o(t) \mid s(t))$ 来强化. 这说明了正强化 (奖励) $\Delta p(o(t) \mid s(t))>0$, 负强化 (惩 罚) $\Delta p(o(t) \mid s(t))<0 . \Delta p(o(t) \mid s(t))$ 是在时刻 $t$ 跟随在操作 性行为 $o(t)$ 后的强化. 尽管在操作性行为和它的强

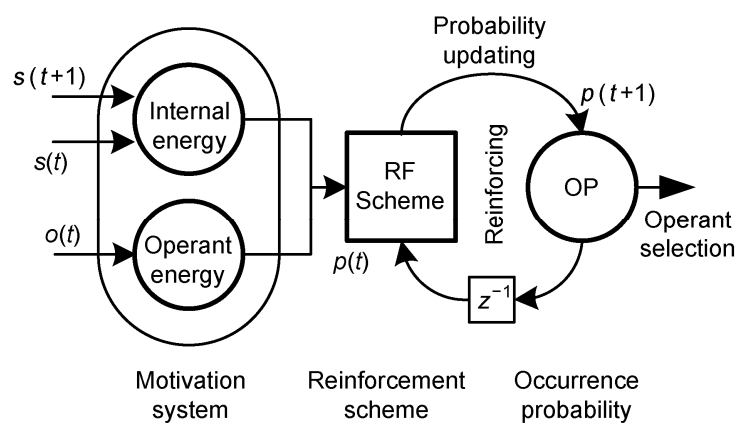

图 3 操作性条件反射中的动机和强化
化刺激之间存在时间差, 图 3 和(6)式中所示的操作 性条件反射的强化遵守因果同时性原理.

从以上的分析看出, 操作性条件反射有 3 个基本 的元素: 1) 操作性行为是主动的或者自发的行为, 2) 能量系统是动机的具体化, 3) 动机系统指导了将操 作性行为发生概率计算地公式化形成的强化.

(2) (6)式分别推广了操作性条件反射中生物的 行为选择, 状态转移, 动机系统和强化, 也成为了我 们设计斯金纳自动机的原则.

\section{3 斯金纳自动机的形成}

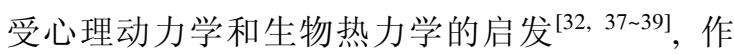
为人工系统 (自主智能体) 以类似动物的操作性条件 反射的方式学习的机器学习的模型, 本节建立了斯 金纳自动机.

\section{1 斯金纳自动机的定义}

从图 1 3 和(2) (6), 斯金纳自动机可以定义为一 个七元组

$$
S A U T O=(t, S, O, M, F, G, A),
$$

其中 $t \in\{0,1,2, \cdots\}$ 代表了离散时间, $S$ 代表内部状态集 合, $O$ 代表操作性行为集合, $M: S(t) \times O(t) \times S(t+1) \rightarrow$ $R^{+}([0,+\infty))$ 代表动机单元, $F: S(t) \times O(t) \rightarrow S(t+1)$ 代表状 态转移过程, $G: S(t) \rightarrow O(t)$ ( $p$ ) 代表操作性行为选择过 程, $A$ 代表斯金纳算法.

在斯金纳自动机中，离散事件具有两重含义：1) 为了数字计算使操作性条件反射的过程离散化, 2) 标示了因(操作性行为)果(结果或强化刺激)的同时 性. 每个离散时间都代表了操作性条件反射过程的 一个阶段.

$F$ 是斯金纳自动机的状态转移过程, 在每个时刻 $t$, 把当前状态 $s(t) \in S$ 和当前操作性行为 $o(t) \in O$ 映射 到下一个状态 $s(t+1) \in S$. 它一般是一个确定性的或者 随机性的黑箱.

$G$ 是斯金纳自动机的操作性行为选择过程, 在 每个时刻 $t$, 把当前状态 $s(t) \in S$ 通过发生概率 $p$ 映射 到当前操作性行为 $o(t) \in O$. 换句话说, 在每个时刻 $t$, 斯金纳自动机在当前状态 $s(t)$ 的基础上选择当前操作 性行为(动作).

$M$ 是斯金纳自动机的动机单元，它是一个以 $\tilde{E}_{\mathrm{S}}$ 
为内能函数, $\tilde{\sigma}_{\mathrm{O}}$ 为操作性行为能量函数的能量系统. 在(4)的基础上, 在每个时刻 $t$, 它将内能增量 $\Delta \mathcal{E}_{S}(t)$ 从 $s(t)$ 映射到 $s(t+1)$, 将操作性行为 $o(t)$ 的能量 $\varepsilon_{0}(t)$ 从 $\Delta \tilde{\mathscr{G}}_{\mathcal{S}}(t)$ 映射到 $R^{+}([0,+\infty))$. 动机单元 $M$ 使斯金纳自动 机渴望用最少的操作性行为能量来保持最低的能量 状态.

\section{2 操作性行为的发生概率}

在操作性条件反射理论中, 生物或者动物趋于 更多地展现导致奖励的行为, 更少地展现导致惩罚 的行为. 这就是说操作性条件反射趋于奖励一个操 作性行为, 如果它的结果和生物趋向性或者动机一 致, 这样它的发生概率就会增大, 反之则惩罚它使概 率减小.

在心理动力学和生物热力学中, 操作性条件反 射是一个热力学过程, 因此可以用热力学的方法来 研究它. 这使我们可以结合以 Monte Carlo 方法为基 础的 Metropolis 算法和模拟退火算法.

Monte Carlo 方法依赖于重复随机采样来计算它 的结果 ${ }^{[41]}$. 它可以用来研究操作性条件反射中斯金 纳自动机的操作性行为和结果的联系. 状态 $s(t)$ 和操 作性行为 $o(t)$ 可以在每个时刻 $t$ 随机选取出来生成下 一个状态 $s(t+1)$. 把所有时刻的结果都加起来可以得 到 $F$ 的统计特性. 然而在 Monte Carlo 方法中操作性 行为和结果之间不存在条件反射.

在统计热力学应用中, Metropolis 和 Jorgensen 等 人 ${ }^{[28,29]}$ 提出了改进的 Monte Carlo 过程来获得构型积 分的实用方法, 其中定义内能函数 $\mathcal{E}$ 用于热力学系统. 他们关键的贡献在于不再无条件地接受目前构型 $s$ 的 随机突变 $s^{\prime}$ 而是以概率接受 $s^{\prime}$

$$
p\left(s^{\prime} \mid s\right)=\left\{\begin{array}{cc}
1, & \Delta \mathscr{E} \leqslant 0, \\
\mathrm{e}^{-\Delta \mathscr{E} / K_{\mathrm{B}} T}, & \Delta \mathcal{E}>0,
\end{array}\right.
$$

其中 $T$ 是温度, $K_{\mathrm{B}}$ 是波尔兹曼常数, 并且 $\Delta \mathscr{E}=\mathscr{E}\left(s^{\prime}\right)-$ $\mathscr{E}(s)$ 是能量增量. (8)式说明突变 $s^{\prime}$ 的内能越低, 它被 接受的概率就越大. 如果 $\Delta \mathscr{G} \leqslant 0, s^{\prime}$ 就一定会被接受. Metropolis 算法保证了产生一个服从波尔兹曼分布的 状态的总体.

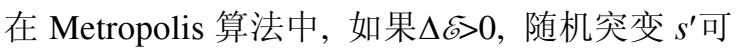
以被拒绝. 然而在操作性条件反射中, 状态 $s(t+1)$ 不 能用 Metropolis 的方式拒绝, 因为它已经成为了操作 性行为 $o(t)$ 的结果. 生物或者动物不能拒绝任何因它
自身行为产生的结果. 它只能试图通过减少相应的 操作性行为的出现频率来避免不好的结果. 和生物 或者动物一样, 如果状态 $s(t+1)$ 比 $s(t)$ 的内能要高并 且 $o(t)$ 的操作性能量比较高, 斯金纳自动机就会通过 操作性条件反射的方式来调节操作性行为 $o(t)$ 在状态 $s(t)$ 下出现的概率使 $s(t+1)$ 出现在 $s(t)$ 之后的机会减小, 反之, 如果内能减小并且 $o(t)$ 的操作性能量比较低, 就增大它的概率. 这里我们定义操作性行为 $o(\in O)$ 在 状态 $s(\in S)$ 下出现的概率为

$$
\left\{\begin{array}{l}
p(o \mid s)=\frac{1}{Z(s)} \mathrm{e}^{-\left\langle\delta_{\mathrm{SYS}}(o \mid s)\right\rangle / K_{\mathrm{B}} T}, \\
Z(s)=\sum_{\theta \in O} \mathrm{e}^{-\left\langle\delta_{\mathrm{SYS}}(\theta \mid s)\right\rangle / K_{\mathrm{B}} T},
\end{array}\right.
$$

其中 $\mathscr{\mathcal { E }}_{\mathrm{SYS}}(o \mid s)=\Delta \mathcal{E}_{\mathrm{S}}(o \mid s)+\mathscr{E}_{\mathrm{O}}(o)$ 是(4)中能量系统的能量 值, $\mathscr{E}_{\mathrm{O}}(o)$ 是 $o \in O$ 的操作性能量, $\Delta \mathscr{E}_{\mathrm{S}}(o \mid s)$ 是有操作性行 为 $o \in O$ 在状态 $s \in S$ 下引起的内能增量. $\langle\bullet\rangle$ 是 $\tilde{\mathcal{S}}_{\mathrm{SYS}}(o \mid s)$ 在 $o$ 在操作性条件反射的状态转移过程中在状态 $s$ 下 反复出现的统计量. (9)和(6)式是一致的, 推广了操作 性条件反射的强化机制. 这说明有更低的操作性能 量并且使斯金纳自动机内能减少的操作性行为趋向 于更大的出现概率.这也和 Metropolis 算法中(8) 类似.

一般地, 状态转移过程 $F$ 可以是一个概率模型, 是一个随机过程, 或者是一个具有不确定性的系统. 我们可以通过统计每个离散时间 $t$ 的能量值 $\tilde{E}_{S Y S}(o \mid s)$ 来得到(9)中的统计量 $\left\langle\mathcal{E}_{S \mathrm{SY}}(o \mid s)\right\rangle$. 例如, $\left\langle\mathcal{E}_{\mathrm{SYS}}(o \mid s)\right\rangle$ 可 以是能量值的算数平均或者加权平均. 一个可选的 统计方法是在 $t$ 时刻, $\forall \bar{o} \in O$ 且 $\bar{s} \in S$, 如果 $\bar{o}=o(t)$ 且 $\bar{s} \in s(t)$, 则

$$
\left\langle\tilde{\mathcal{G}}_{\mathrm{SYS}}(\bar{o} \mid \bar{s})\right\rangle=\sigma \tilde{\mathcal{E}}_{\mathrm{SYS}}(o(t) \mid s(t))+(1-\sigma)\left\langle\tilde{\mathcal{E}}_{\mathrm{SYS}}(\bar{o} \mid \bar{s})\right\rangle,
$$

其中 $\sigma \in(0,1)$. (10)式实际上是一个低通滤波器, 有 双重含义: 1) $\left|\left\langle\varepsilon_{\mathcal{S}_{\mathrm{SS}}}(\bar{o} \mid \bar{s})\right\rangle\right|$ 通过操作性条件反射的 迭代从零逐渐成为一个相对稳定的统计量, 2) 对环 境的改变有一定的适应性.

\section{3 斯金纳算法}

斯金纳算法是斯金纳自动机的核心, 它模拟了 操作性条件反射的机制, 在斯金纳自动机中相当于 强化策略. 它从 Metropolis Monte Carlo 方法 ${ }^{[28,29]}$ 和 模拟退火算法 ${ }^{[30,31]}$ 行生出它的基本原理. 通过斯金 
纳算法, 斯金纳自动机可以像热力学系统一样运行, 并且试图获得一些最优学习.

斯金纳算法运用 Monte Carlo 方法在斯金纳自动 机每个时刻 $t$ 的状态转移过程中重复地对操作性行为结果进行采样并且把操作性行为的效果累加. 它用和 (8)中 Metropolis 算法一致的的方式通过能量函数定义 了操作性行为的发生概率. 另外, 它通过 Metropolis 算法建立了斯金纳自动机的操作性条件反射. 这说 明斯金纳自动机在特定温度达到了热平衡. 然而, 条 件反射式的操作性行为不一定是全局最优的.

受到冶金工业中退火的启发, Kirkpatrick 等人 ${ }^{[30]}$ 在 1983 提出了用于全局优化的模拟退火算法, Černý ${ }^{[31]}$ 于 1985 年在 Metropolis 算法中引入温度机制. 模拟退火算法在一个很高的温度启动, 然后逐渐冷 却，这样搜索空间就会逐步收缩，最终成为一个小的 具有全局能量最小的状态集合. 事实上, 动物的学习 和退火都是逐渐优化的过程. 操作性条件反射是一 个行为选择的过程, 或者也可以说是一个行为优化 的过程. 开始的时候, 对于每个状态, 操作性学习都 有一个较大的可选操作性行为的集合, 每个状态又 可能包括多个状态, 其中也有高能量状态. 这与高温 状态下的退火类似. 逐渐地, 一些操作性行为因为与 生物趋向性或者动机相违背, 它们将不再被选择. 最 终, 可选的操作性行为集合收缩为一个具有最优行 为的小集合. 很明显模拟退火算法和操作性条件反 射具有相似的特性. 最值得注意的是模拟退火算法 中的降温可看作操作性条件反射中的强化过程. 根 据(9), 一个好的操作性行为可以使 $F$ 的内能降低, 在 降温过程中, 它也会得到更多出现的机会.

斯金纳算法有基本的 5 个步骤.

1) 初始化: $\forall s \in S$ 且 $o \in O$, 令 $\left\langle\tilde{E}_{S Y S}(o \mid s)\right\rangle=0$. 令初 始时间 $t$ 为 0 , 初始状态 $s(t)=s_{0}(\in S)$, 人工温度 $T_{A}$ $\left(=K_{\mathrm{B}} T\right)$ 足够高.

2) 行为选择: 通过 $(9)$ 和 $\left\langle\tilde{E}_{S Y S}(o \mid s(t))\right\rangle$, 在状态 $s(t)$ 计算操作性行为的出现概率, 并通过概率从 $O$ 中选 择操作性行为 $o$.

3) 执行操作性行为: 将操作性行为 $o(t)=o$ 代入 状态转移过程 $F$ 得到下一个状态 $s(t+1)$.

4) 将操作性行为的效果累加: 得到由 $o(t)$ 和 $s(t)$ 作用产生的能量值 $\sigma_{S Y S}(o(t) \mid s(t))$, 求得能量系统的统 计量 $\left\langle\tilde{E}_{S Y S}(o(t) \mid s(t))\right\rangle$. 令 $t=t+1$, 重复第二步, 直到在温 度 $T_{\mathrm{A}}$ 达到热平衡或者重复的次数足够多.
5) 通过降温强化: 根据特定的降温策略降低温 度 $T_{A}$, 从第二步重复直到 $T_{A}$ 足够低或者 $F$ 达到冻结 状态.

斯金纳算法开始时, $t=0$, 对 $S$ 中任意状态, 所有 的 $O$ 中的操作性行为的出现概率是相等的. 然而到 了最后, 只会有很少的几个操作性行为被激发来维 持最低能量的状态.

斯金纳算法可以用 Metropolis Monte Carlo 方法 和模拟退火的方式模拟操作性条件反射以及行为选 择过程.

\section{4 热平衡和操作性行为熵}

斯金纳自动机是一个热力学系统, 也是一个自 治的心理动力学系统. 它的自治特性通过它的热平 衡现象体现.

为了展示斯金纳自动机的热平衡现象，我们假 设一个相对简单的情形, $F$ 是确定性的, $\left\langle\Delta E^{(S)}(\boldsymbol{o} \mid \boldsymbol{s})\right\rangle$ 是 内能增量的均值. 那么(5)就有如下的形式:

$$
\left\{\begin{array}{l}
p(\boldsymbol{o} \mid s)=\frac{1}{Z(\boldsymbol{s})} \mathrm{e}^{-E^{(s)}(\boldsymbol{o} \mid \mathbf{s}) / K_{\mathrm{B}} T}, \\
Z(\boldsymbol{s})=\sum_{\boldsymbol{\theta} \in O} \mathrm{e}^{-E^{(s)}(\boldsymbol{\theta} \mid s) / K_{\mathrm{B}} T},
\end{array}\right.
$$

其中 $E^{(S)}(\boldsymbol{o} \mid \boldsymbol{s})=E^{(S)}\left(\boldsymbol{s}^{\prime}\right)$ 是由 $\boldsymbol{o}$ 在 $\boldsymbol{s}$ 下引起状态 $\boldsymbol{s}^{\prime}$ 的内能. (11)中, 在给定状态下, 任意操作性行为的发生概率 都与波尔兹曼因子成比例 ${ }^{[40]}$,

$$
p(\boldsymbol{o} \mid \boldsymbol{s}) \propto \mathrm{e}^{-E^{(S)}(\boldsymbol{o} \mid s) / K_{\mathrm{B}} T} .
$$

考虑一种理想情况, $\forall \boldsymbol{s}$ 且 $\boldsymbol{s}^{\prime} \in S$, 存在一个互斥的 $\boldsymbol{o} \in O$ 使 $F$ 从 $\boldsymbol{s}$ 到 $\boldsymbol{s}^{\prime}$. 那么从(11), $\forall \boldsymbol{s}_{\alpha}$ 且 $\boldsymbol{s}_{\beta} \in S$, 存在 $\boldsymbol{o}_{\alpha}$ 和 $\boldsymbol{o}_{\beta} \in O$ 使

$$
\frac{p\left(\boldsymbol{s}_{\beta}\right)}{p\left(\boldsymbol{s}_{\alpha}\right)}=\frac{p\left(\boldsymbol{s}_{\alpha} \rightarrow \boldsymbol{s}_{\beta}\right)}{p\left(\boldsymbol{s}_{\beta} \rightarrow \boldsymbol{s}_{\alpha}\right)}=\frac{p\left(\boldsymbol{o}_{\alpha} \mid \boldsymbol{s}_{\alpha}\right)}{p\left(\boldsymbol{o}_{\beta} \mid \boldsymbol{s}_{\beta}\right)}=\mathrm{e}^{-\left(E\left(s_{\beta}\right)-E\left(s_{\alpha}\right)\right) / K_{\mathrm{B}} T},
$$

其中 $\boldsymbol{s}_{\alpha} \rightarrow \boldsymbol{s}_{\beta}$ 代表了从 $\boldsymbol{s}_{\alpha}$ 到 $\boldsymbol{s}_{\beta}$ 的状态转移过程. (13)式意 味着在 ${ }^{[36,40]}$ 中, 斯金纳自动机的状态服从波尔兹曼吉布斯分布, 并且会在特定温度 $T$ 达到热平衡.

一般地, 状态转移过程 $F$ 是一个概率模型, 一个 随机过程或者是具有不确定性的物理系统. 取 (5)和 (6)中每个离散时间 $t$ 的能量增量 $\Delta E^{(S)}$ 来计算统计量 $\left\langle\Delta E^{(S)}\right\rangle$ 一个可选的统计方法是 


$$
\left\langle\Delta E_{t+1}^{(S)}\left(\boldsymbol{o}_{t} \mid s_{t}\right)\right\rangle=\sigma \Delta E_{t}^{(S)}\left(\boldsymbol{o}_{t} \mid s_{t}\right)+(1-\sigma)\left\langle\Delta E_{t}^{(S)}\left(\boldsymbol{o}_{t} \mid s_{t}\right)\right\rangle,
$$

其中 $\sigma \in(0,1),\left\langle\Delta E_{t}^{(S)}\right\rangle$ 是 $\left\langle\Delta E^{(S)}\right\rangle$ 在 $t$ 时刻的值, $\Delta E_{t}^{(S)}\left(\boldsymbol{o}_{t} \mid \boldsymbol{s}_{t}\right)=E^{(S)}\left(\boldsymbol{s}_{t+1}\right)-E^{(S)}\left(\boldsymbol{s}_{t}\right)$ 是 $\boldsymbol{o}_{t}$ 在 $t$ 时引起的 内能增量. (14)式实际上是一个低通滤波器, 有两重 含义: 1) $\left|\left\langle\Delta E_{t}^{(S)}\right\rangle\right|$ 通过重复的操作性条件反射从零 逐步成为稳定的统计量, 2) 对于环境的改变有一定 的适应能力.

熵的概念是从热力学第二定律得出, 被推广为 系统无序度的量度. 这里我们引入斯金纳自动机的 操作性熵的概念来描述斯金纳自动机中操作性行为 的不确定性

$$
H=-\sum_{\boldsymbol{s} \in S} \sum_{\boldsymbol{o} \in O} p(\boldsymbol{o} \mid s) \ln p(\boldsymbol{o} \mid \boldsymbol{s}) .
$$

事实上, 操作性熵就是行为熵 ${ }^{[41]}$, 用来描述人 或动物或自主智能体的行为的不确定性.

很明显, 斯金纳自动机的操作性熵总是随着温 度的下降而下降, 最终收玫为 0 . 这说明在斯金纳自 动机中, 操作性条件反射是一个自治的过程.

\section{5 饥饿的斯金纳鸽子}

从某种意义上说，操作性条件反射是由斯金纳 做的一系列鸽子实验而来. 斯金纳进行了大量的用 鸽子做的实验来展示他的操作性条件反射理论 ${ }^{[4]}$. 他 设计了一套隔音装置, 通常称为斯金纳箱(图 4), 用 它来进行操作性条件反射的实验. 斯金纳箱具有食 物托盘和布撒器, 有 3 种颜色的按钮(红, 蓝, 黄)来 让鸽子豚. 鸽子啄红色按钮会得到食物, 蓝色会遭到 电击, 黄色什么都不会发生. 起初, 鸽子随机地啄按 钮. 然而通过操作性条件反射, 它逐渐学会了只豚红 色按钮.

像斯金纳鸽子一样, 斯金纳自动机也可以学会

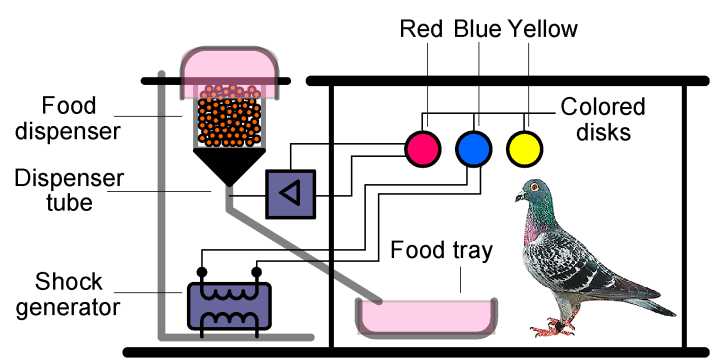

图 4 斯金纳箱和其中的鸽子

豚正确的按钮来得到它想要的. 这里我们展示用斯 金纳自动机来重现斯金纳鸽子实验. 斯金纳自动机 用来模拟斯金纳箱中的鸽子. 模拟的鸽子豚红色按 钮得到食物, 蓝色遭到电击, 黄色什么也没有.

作为一个例证，斯金纳自动机模拟的鸽子很小 也很简单. 它的状态转移过程 $F$ 在图 5 和表 1 中展示, 只有 3 个状态. 1) $s^{(0)}$ : 满足. 2) $s^{(1)}$ : 期盼食物. 3) $s^{(2)}$ : 痛苦. 对状态 $\boldsymbol{s}^{(1)}$ 有 3 个可选的操作性行为. 1) $\boldsymbol{o}^{(1)}$ : 豚 红色按钮. 2) $\boldsymbol{o}^{(2)}$ : 啄黄色按钮. 3) $\boldsymbol{o}^{(3)}$ : 啄蓝色按钮. 只有一个操作性行为 $\boldsymbol{o}^{(0)}$ 在状态 $s^{(0)}$ 和 $s^{(2)}$ 下是什么都 不做, 这样就可以自动回到状态 $\boldsymbol{s}^{(1)}$. 内能函数定义 为 $E^{(S)}\left(\boldsymbol{s}^{(k)}\right)=k^{2}$, 例如, 1) $\left.\left.E^{(S)}\left(\boldsymbol{s}^{(0)}\right)=0,2\right) E^{(S)}\left(\boldsymbol{s}^{(1)}\right)=1,3\right)$ $E^{(S)}\left(s^{(2)}\right)=4$. 状态 $s^{(0)}$ 是模拟鸽子期望的能量最低状态. 没有考虑操作性行为能量, 即 $\forall \boldsymbol{o} \in O$ 使 $E^{(O)}(\boldsymbol{o})=0$.

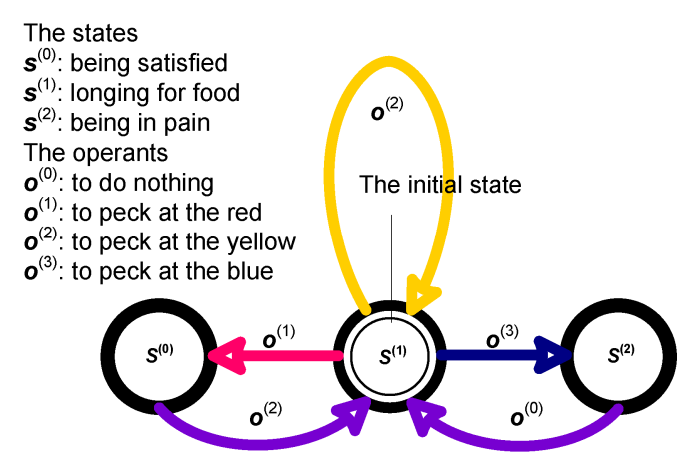

图 5 斯金纳自动机模拟的鸽子的状态转移图

\section{表 1 模拟的鸽子的状态转移表}

\begin{tabular}{lccc}
\hline & $\boldsymbol{s}^{(0)}:$ being satisfied & $\boldsymbol{s}^{(1)}$ : longing for food & - \\
\hline $\boldsymbol{o}^{(0)}:$ to do nothing & $\boldsymbol{s}^{(1)}$ & $\boldsymbol{s}^{(0)}$ being in pain & $\boldsymbol{s}^{(1)}$ \\
$\boldsymbol{o}^{(1)}:$ to peck at the red & - & $\boldsymbol{s}^{(1)}$ & - \\
$\boldsymbol{o}^{(2)}:$ to peck at the yellow & - & $\boldsymbol{s}^{(2)}$ & - \\
$\boldsymbol{o}^{(3)}:$ to peck at the blue & - & - \\
\hline
\end{tabular}


模拟实验的结果如图 6 和 7 所示. 初始人工温度 $T_{A}$ 是 $10000^{\circ} \mathrm{C}$, 降温过程为

$$
T_{\mathrm{A}}(k+1)=\sigma T_{\mathrm{A}}(k),
$$

其中 $\sigma=0.9$.

起初, 如图 6 所示, 斯金纳自动机模拟的鸽子在 状态 $s^{(1)}$ 豚每个按钮的概率相等, 为 $1 / 3$. 在操作性学 习的冷却过程中, 斯金纳自动机为了获得食物, 豚红 色按钮的次数越来越多. 通过操作性条件反射, 斯金 纳自动机的操作性行为 $\boldsymbol{o}^{(1)}$ 的发生概率逐渐从 $1 / 3$ 增 加到 1 , 另外两个操作性行为 $\boldsymbol{o}^{(2)}$ 和 $\boldsymbol{o}^{(3)}$ 的发生概率逐 渐从 $1 / 3$ 降为 0 . 有趣的是, 为了避免遭受电击, 豚蓝 色按钮的概率下降得比豚黄色按钮要快得多. 通过 实验中的操作性条件反射, 模拟的斯金纳鸽子通过 斯金纳自动机学会了豚正确的按钮来获得食物, 并 且避免豚不正确的按针.

图 7 显示斯金纳自动机的内能均值随着温度降 低也越来越低. 同时它的操作性熵也在逐步降低. 这说明斯金纳自动机模拟的鸽子的操作性学习是自 治的.

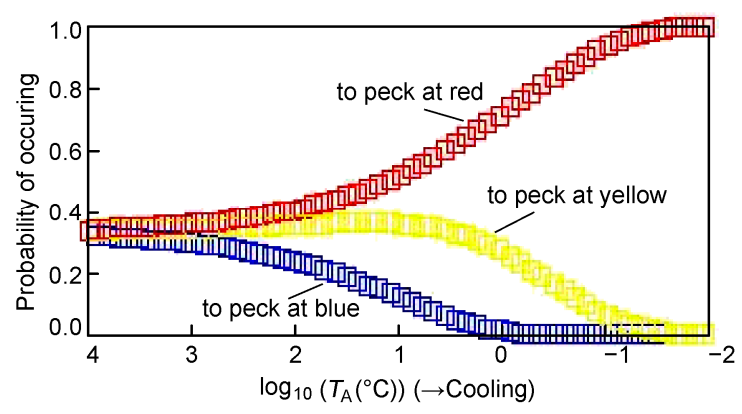

图 6 操作性行为概率图

状态 $s^{(1)} 3$ 个可选操作性行为的发生概率, $T_{\mathrm{A}}\left(=K_{\mathrm{B}} T\right)$ 是人工温度

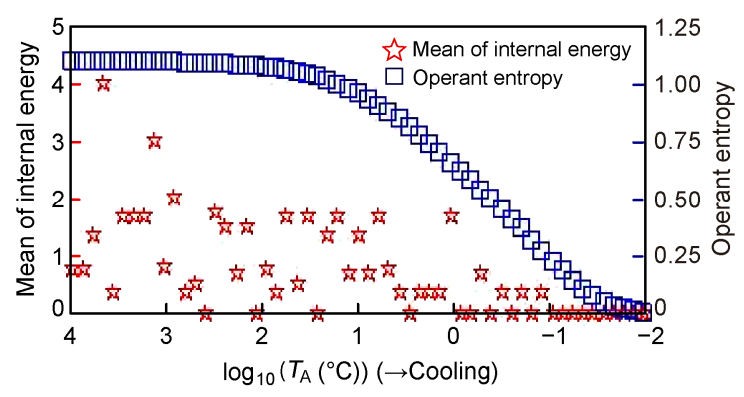

图 7 操作性摘和内能均值 $\left(T_{\mathrm{A}}\left(=K_{\mathrm{B}} T\right)\right.$ 是人工温度 $)$
在每个模拟试验中, 模拟鸽子在温度从 $10000^{\circ} \mathrm{C}$ 降到 $0.011^{\circ} \mathrm{C}$ 的过程中需要豚按钮大约 2000 次来实现 操作性学习. 在一个模拟实验中, 模拟鸽子啄了 1885 次按钮, 其中 1115 次红色, 445 次黄色, 325 次蓝 色. 我们假设斯金纳自动机的离散时间间隔是 $15 \mathrm{~s}$, 这就花费了鸽子大约 8 个小时来完成操作性学习.

\section{6 斯金纳鸽子为什么迷信}

斯金纳在迷信鸽子上做的实验很有趣 ${ }^{[42]}$. 他发 现鸽子会迷信. 为了祈求食物, 斯金纳鸽子会展示奇 怪的行为, 如拍翅膀、转圈以及扭脖子, 这些都是所 谓的迷信行为. 如果动作时间和食物投放时间吻合, 斯金纳鸽子趋向于加强它们的行为, 然而食物的投 放完全和它们的行为无关.

为什么鸽子会迷信呢? 斯金纳自动机也许可以 重现这个实验并指引我们理解它. 就像斯金纳鸽子 一样，斯金纳自动机也会迷信.

我们用斯金纳自动机模拟了几个鸽子来展示为 什么斯金纳鸽子会迷信. 如图 8, 我们设计了一个模 拟的斯金纳箱, 有食物托盘和定时器. 每 15 分钟布 撒器会投放一粒食物. 食物的投放与斯金纳自动机 模拟的鸽子的行为没有任何关系. 然而就像斯金纳 鸽子一样, 斯金纳自动机也会加强与食物投放时间 相吻合的动作. 我们将迷信行为称作伪操作, 根据强 化伪操作, 称为伪操作性条件反射.

斯金纳自动机模拟的迷信鸽子的状态转移图如 图 9, 状态表见表 2. 在状态转移图中, 只有两个状态. 1) $s^{(0)}$ : 满足. 2) $s^{(1)}$ : 期盼食物. 模拟鸽子在状态 $s^{(0)}$ 和 $\boldsymbol{s}^{(1)}$ 都有一个操作性行为 $\boldsymbol{o}^{(0)}$ 是什么都不做, 在状态 $\boldsymbol{s}^{(1)}$, 有 6 个伪操作可以发生. 1) $\boldsymbol{o}^{(1)}$ : 拍翅膀. 2) $\boldsymbol{o}^{(2)}$ : 旋转. 3) $\boldsymbol{o}^{(3)}$ : 扭动身体. 4) $\boldsymbol{o}^{(4)}$ : 转脖子. 5) $\boldsymbol{o}^{(5)}$ : 叫.

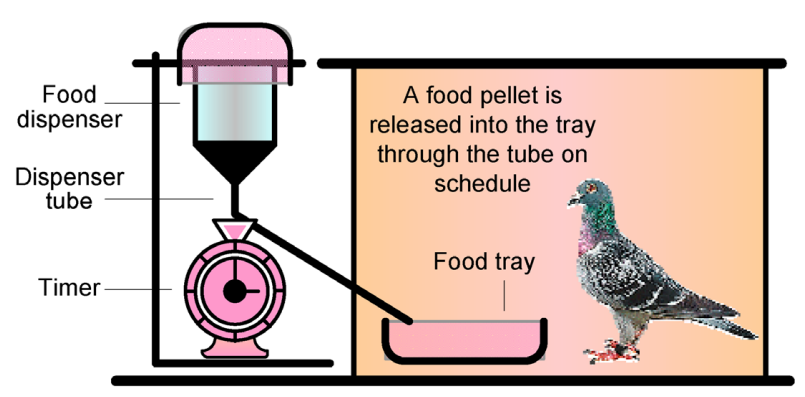

图 8 用模拟斯金纳箱来展示斯金纳迷信鸽子实验 


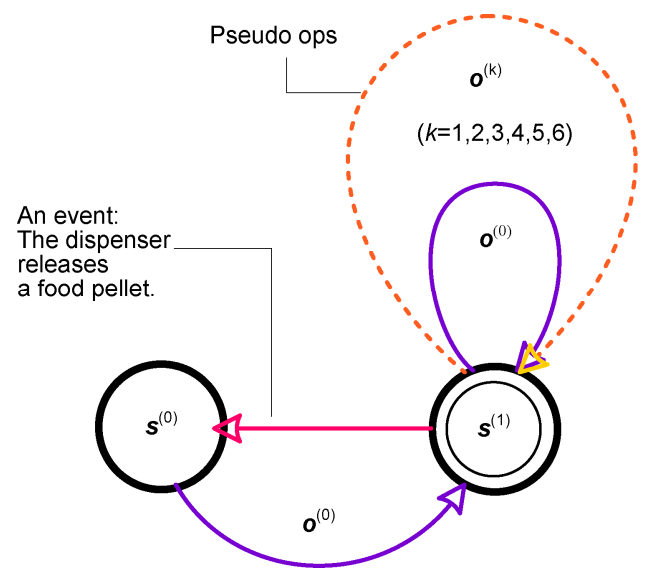

图 9 斯金纳自动机模拟的迷信鸽子的状态转移图

\section{表 2 模拟迷信鸽子的状态转移过程}

\begin{tabular}{|c|c|c|}
\hline & $\boldsymbol{s}^{(0)}$ : being satisfied & $s^{(1)}$ : longing for food \\
\hline $\boldsymbol{o}^{(0)}$ : to do nothing & $\boldsymbol{s}^{(1)}$ & $s^{(1)}$ \\
\hline $\boldsymbol{o}^{(1)}$ : to flap wings & - & $\boldsymbol{s}^{(1)}$ \\
\hline $\boldsymbol{o}^{(2)}:$ to spin & - & $\boldsymbol{s}^{(1)}$ \\
\hline $\boldsymbol{o}^{(3)}$ : to twist & - & $\boldsymbol{s}^{(1)}$ \\
\hline $\boldsymbol{o}^{(4)}:$ to turn the neck & - & $\boldsymbol{s}^{(1)}$ \\
\hline $\boldsymbol{o}^{(5)}:$ to sing & - & $\boldsymbol{s}^{(3)}$ \\
\hline $\boldsymbol{o}^{(6)}$ : to bow & - & $\boldsymbol{s}^{(1)}$ \\
\hline The outside event ${ }^{\#}$ & - & $s^{(0)}$ \\
\hline
\end{tabular}

\#布撒器每 15 分钟投放一次食物, 可能会与操作性行为 $o(k)$ $(0 \leqslant k \leqslant 6)$ 同时发生

6) $\boldsymbol{o}^{(6)}$ : 低头.

模拟的斯金纳鸽子有相同的状态转移图, 但是 内能函数, 操作性行为能量, 以及表现不同心理和生 理状态, 生物趋向性, 认知和学习的采样不同.

\section{1 均衡的操作性条件反射}

第一个模拟鸽子是均衡的并且通过了充分的操 作性学习. 它有内能函数 $E^{(S)}\left(\boldsymbol{s}^{(i)}\right)=i(i=0,1)$ 以及操作性 行为能量函数 $E^{(O)}\left(\boldsymbol{o}^{(k)}\right)=0.5 k(0 \leqslant k \leqslant 6)$. 在操作性条件 反射过程中, 它在每个温度取样 25 个操作性行为-结 果的数据对.

如图 10(a)所示, 通过均衡的操作性学习, 模拟 鸽子学会了什么都不做等待食物. 这说明均衡的模 拟鸽子会学到事实.

\section{2 归于过度活动的伪操作性条件反射}

第 2 只模拟鸽子是过度活动的. 它具有能内函数 $E^{(S)}\left(\boldsymbol{s}^{(i)}\right)=i(i=0,1)$ 和操作性行为能量函数 $E^{(O)}\left(\boldsymbol{o}^{(k)}\right)=$
$-0.5 k(0 \leqslant k \leqslant 6)$, 说明模拟鸽子精力过剩。

如图 10(b)所示, 过度活动的模拟鸽子进入了伪 操作性条件反射的过程, 并且开始迷信. 为了祈求食 物, 它低头. 看来精力过剩和过度活动的鸽子趋向于 迷信.

\section{3 归于欲望过强的伪操作性条件反射}

斯金纳给 8 只鸽子喂的食物少于正常水平, 这样 它们就会饥饿. 然后他把它们放进如图 8 所示的斯金 纳箱. 几天后，他发现其中 6 只开始迷信.

我们用斯金纳自动机模拟了 8 只鸽子. 他们有相 同的内能函数 $E^{(S)}\left(\boldsymbol{s}^{(i)}\right)=25 i(i=0,1)$ 就是说他们都欲望 过强, 以及相同的操作性行为能量函数 $E^{(O)}\left(\boldsymbol{o}^{(k)}\right)=$ $0.5 k(0 \leqslant k \leqslant 6)$. 它们在每个操作性行为-结果阶段只取 样一个数据对, 这意味他们是有偏见的, 并且缺乏足 够的认知和学习.

然而，模拟的鸽子有不同的行为，这是因为操作 性条件反射的模拟实验在计算上是随机过程. 如图 11 所示, 模拟鸽子展现了不同的行为. 在 8 只模拟鸽 子中, 6 只因为伪操作性条件反射迷信. 为了祈求食 物, 两只拍翅膀 (见图 11(a), (b)), 两只转圈 (见图 11(c), (d)), 一只扭动脖子(见图 11(e)), 一只低头(见 图 11(f)), 剩下的什么都不做(见图 11(g), (h)). 如实
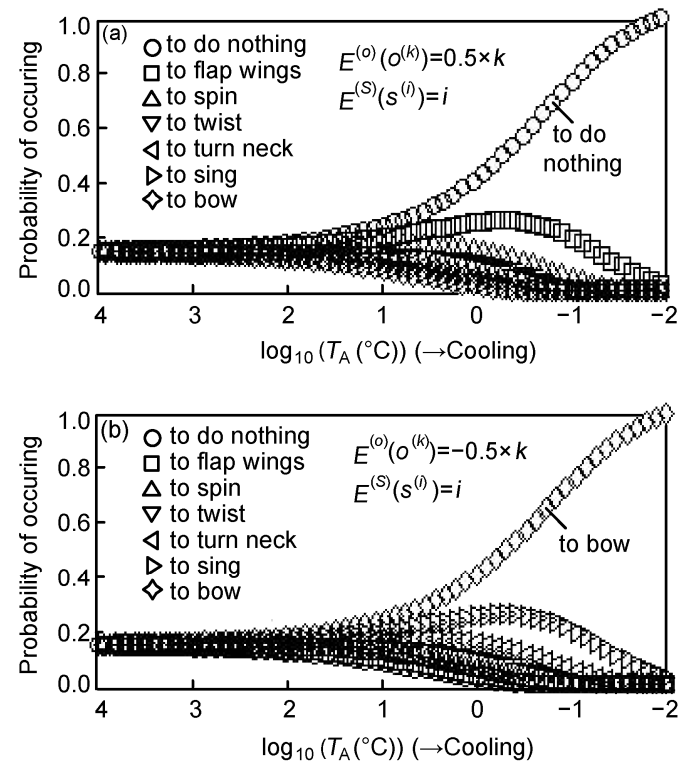

图 10 操作性行为概率图

(a) 均衡的模拟鸽子的学习结果; (b) 过度活动的模拟鸽子的 学习结果 

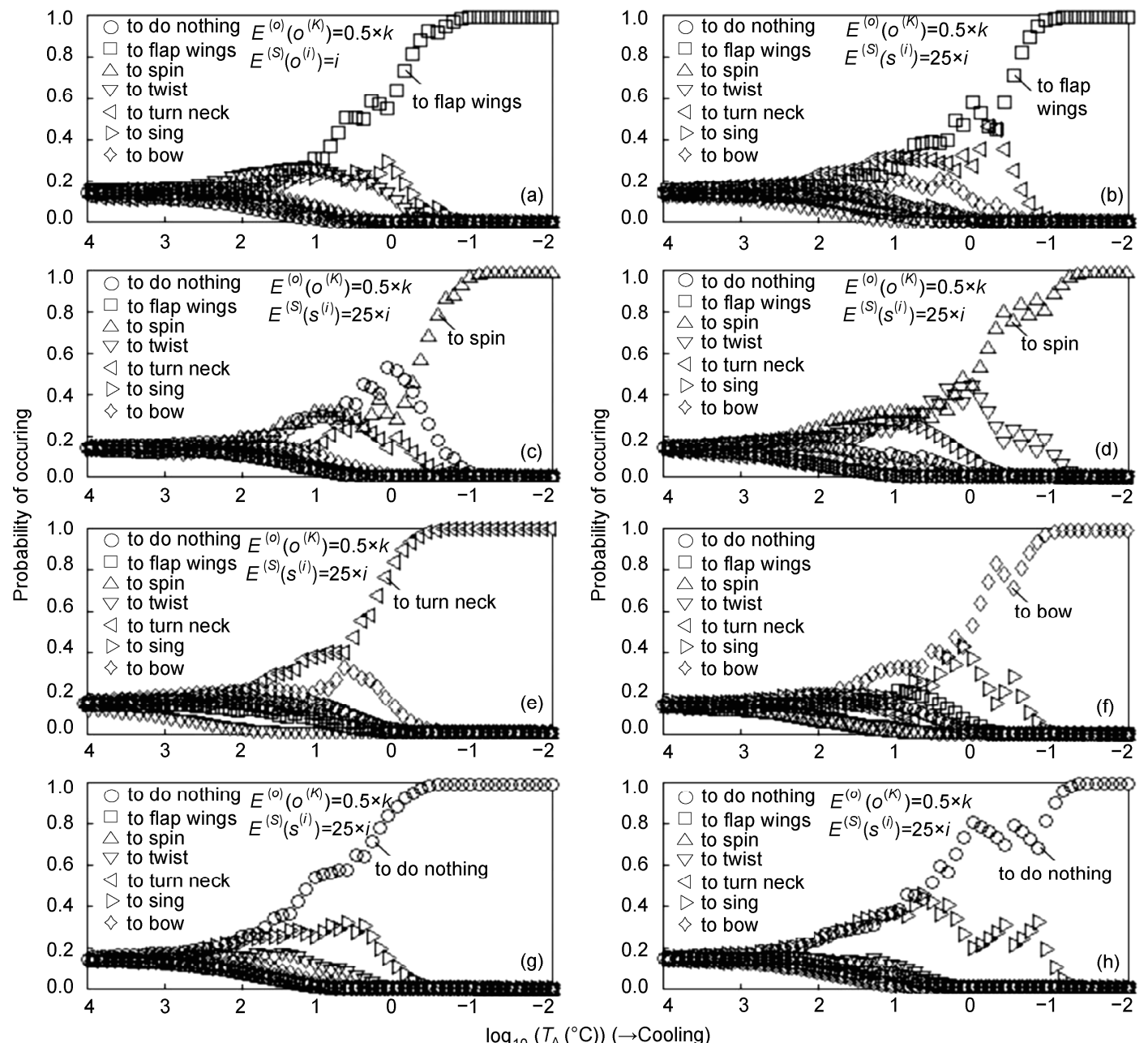

图 11 操作性行为的概率图

从存在偏见和欲望过强的模拟鸽子获得的结果, (a)和(b)是拍打翅膀; (c) 和 (d) 是旋转; (e) 是扭脖子; (f) 是低头; (g)和 (h) 是什么都不做

验中所展示, 状态 $s^{(1)}$ 的能量越高, 取样越少, 模拟鸽 子就越趋于迷信.

我们从斯金纳自动机模拟的迷信鸽子获得了 3 个重要的启发：1）过度活动会导致迷信. 2) 欲望过 强会导致迷信. 3) 偏见以及缺乏学习和认知会导致 迷信.

\section{$73 D$ 维纳虫}

Wiener ${ }^{[43]}$ 作为控制理论之父, 对使机器可以像 动物一样工作很感兴趣. 他在他的书《控制论》中描 述了一个思想实验, 并且构思了模拟负趋光虫子的 机制, 我们随后命名为维纳虫. Wiener 假设电桥可以
作为趋光性动作系统, 这样维纳虫就可以像一只真 正的负趋光的虫子一样搜索黑暗的地方. 受 Wiener 的影响, Braitenberg ${ }^{[44]}$ 也设计了一系列思想实验, 其 中一个具有简单内部结构的小车会以不可预测的复 杂方式行动, 包括趋光的和负趋光的. 维纳虫和 Braitenberg 的小车都很简单但是都很有意义, 因为他 们展示了机器有可能像动物一样行动. 然而 Wiener 和 Braitenberg 的机制只是外在的像动物, 不是内在 的. 它们的行为是被设计好的, 而不是通过自主发展, 认知和学习形成的.

斯金纳自动机可以作为自主智能体以及机器人 的感觉运动系统, 这样可以逐步发展它们像动物一 样的运动技能. 我们设计了如图 12 所示的在虚拟环 

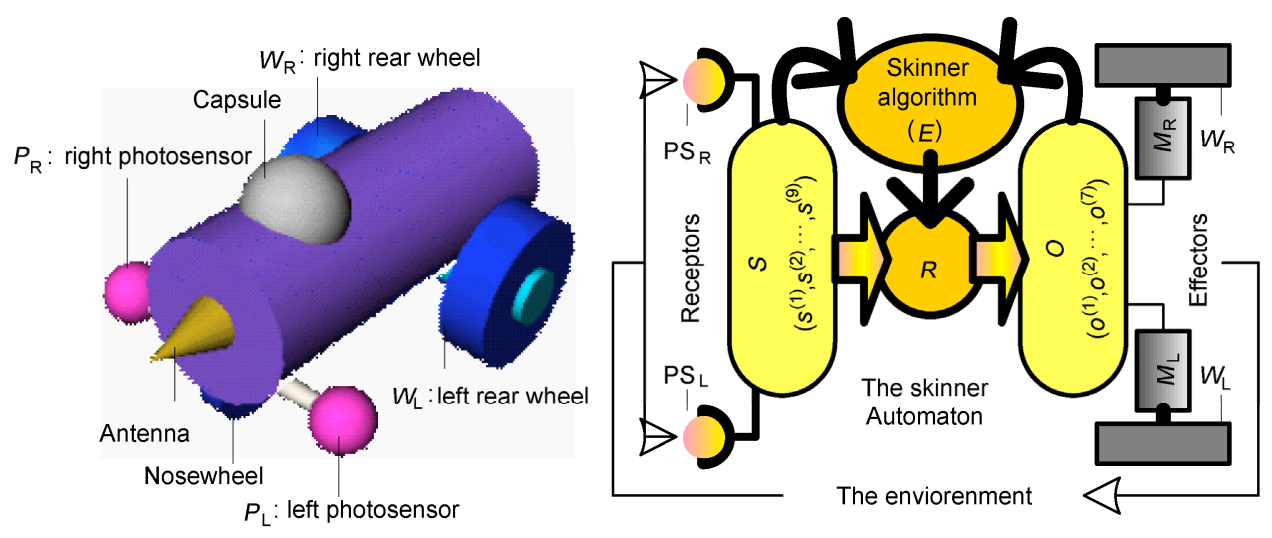

图 12 (a) 3D 维纳虫; (b) 维纳虫的感觉运动系统

境中的 3D 维纳虫. 它 $4 \mathrm{~cm}$ 长, $2.4 \mathrm{~cm}$ 宽, 有两个光 线传感器 $P S_{\mathrm{L}}$ 和 $P S_{\mathrm{R}}$, 以及两个动作电机 $M_{\mathrm{L}}$ 和 $M_{\mathrm{R}}$ 分别驱动左轮 $W_{\mathrm{L}}$ 和右轮 $W_{\mathrm{R}}$.

如图 12(b)所示, 斯金纳自动机作为维纳虫的感 光动作系统, 接收传感器 $P S_{\mathrm{L}}$ 和 $P S_{\mathrm{R}}$ 的信号, 给 $M_{\mathrm{L}}$ 和 $M_{\mathrm{R}}$ 发送操作性行为信号. 斯金纳自动机的每个状 态都是一个向量 $\boldsymbol{s}=(\Delta \rho, \bar{\rho})$, 其中 $\Delta \rho=1$ 如果 $\rho_{\mathrm{L}}>\rho_{\mathrm{R}}$, 否则 $\Delta \rho=-1, \rho_{\mathrm{L}}$ 和 $\rho_{\mathrm{R}}$ 是 $P S_{\mathrm{L}}$ 和 $P S_{\mathrm{R}}$ 测量到的光强. 并 且 $\bar{\rho}=\left(\rho_{\mathrm{L}}+\rho_{\mathrm{R}}\right) / 2$ 被分为 9 个等级. 因此斯金纳自动 机总共有 18 个状态分级. 内能函数定义如下

$$
E^{(S)}(s)=250 \times \bar{\rho}^{2} .
$$

斯金纳自动机的每个操作性行为都是一个向量 $\boldsymbol{o}=(\psi, v)$, 其中 $v$ 是速度的启发性信号, $\psi$ 是方向的启 发性信号; $v$ 分为 4 个等级, $0,1,2,3$. 如果 $v=0$, 那么 $\psi=0$, 否则 $\psi=0.157 \times \Delta \rho$. 所以维纳虫共有 7 个可选的 操作性行为. 操作性行为能量函数定义如下

$$
E^{(O)}(\boldsymbol{o})=0.025 \times v, \quad(v \in\{0,1,2,3\}) .
$$

维纳虫被限制在一个直径 $26 \mathrm{~cm}$ 的圆形场地中, 场地中间有光源, 这样场地的中间是明亮的, 周边黑 暗, 如图 13. 维纳虫的操作性学习过程如图 14 所示, 当维纳虫离墙壁过近时, 壁障机制会启动. 通过操作 性学习, 维纳虫逐渐发展出了它的负趋光的运动技 能. 图 14 展示了维纳虫在不同温度时的运动轨迹. 在 $T_{A}=10000^{\circ} \mathrm{C}$ 时用了 3000 个离散时间间隔, 其他温 度是 1000 个时间间隔. 图 14 说明随着斯金纳自动机 的降温, 维纳虫的自组织程度越来越高. 维纳虫在 $T_{A}=10000^{\circ} \mathrm{C}$ 时是完全无组织的, 但是在 $T_{A}=0.001^{\circ} \mathrm{C}$

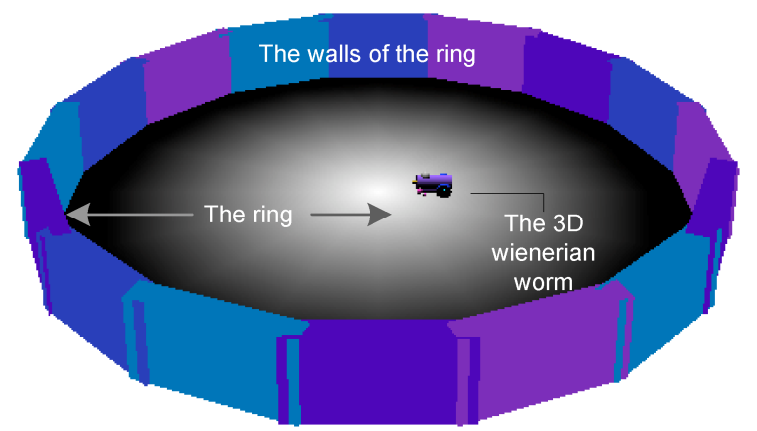

图 13 维纳虫所在的场地

时是高度自组织的. 完成操作性学习后, 维纳虫可以 熟练地搜索黑暗的环境, 并且从任意起点出发都能 到达黑暗的地方, 最终它在墙角缓慢移动或停止.

\section{8 学习平衡技能的机器人}

两轮机器人是一类平衡机器人, 与生俱来的动 力学不稳定性使他们吸引人. 这种机器人的特征是 通过两个轮子来保持平衡以及在原地自旋的能力 ${ }^{[45]}$, 但是这些能力都是人为设计或者事先安排好的.

从某种意义上说, 两轮机器人是一类仿生系统, 它们模仿人类的直立姿态并且展现平衡技能来保持 平衡的姿态. 人类的平衡技能是通过操作性学习来 发展和形成的, 其中操作性条件反射扮演了重要的 角色. 冊庸置疑机器人可以像人类和动物一样学习 是具有意义的.

我们建立了两轮机器人的物理系统 ${ }^{[46]}$, 称为原 人 3 号, 它 $58 \mathrm{~cm}$ 高, $22.5 \mathrm{~kg}$ 重(见图 $15(\mathrm{a})$ ). 原人 3 号是具有复杂动力学特性的柔性平衡机器人. 它有 


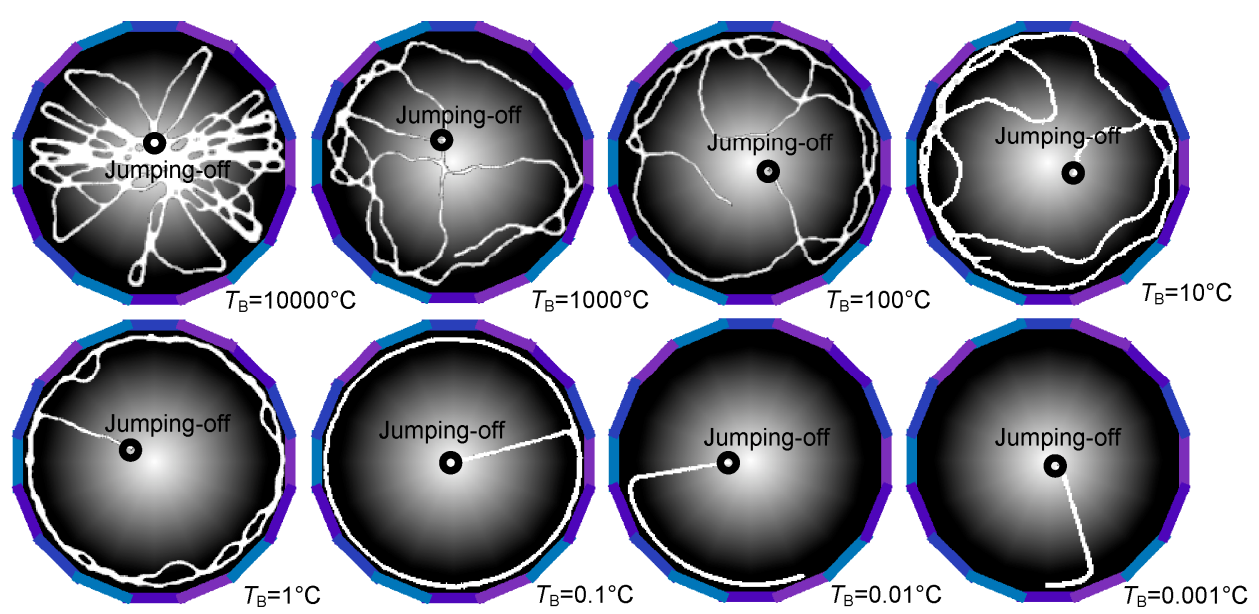

图 14 操作性学习过程中维纳虫的运动轨迹

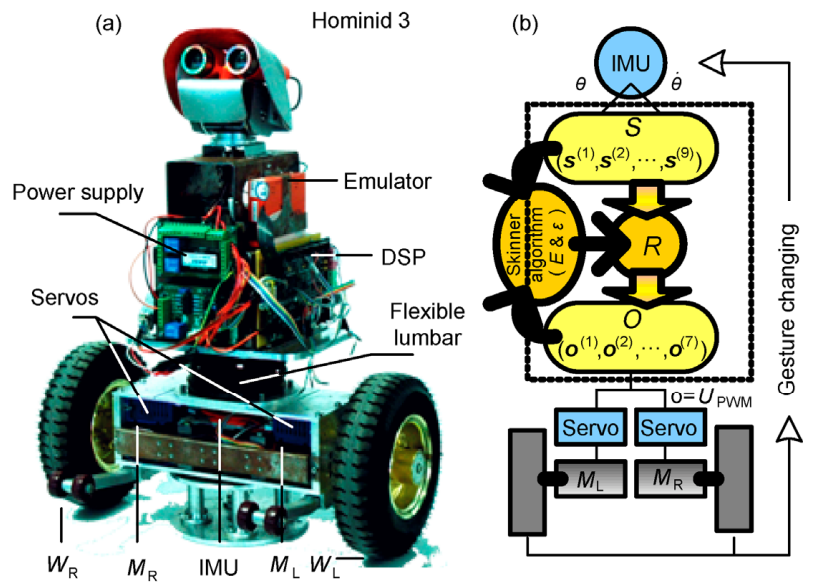

图 15 原人 3 号

(a) 机器人的物理构形; (b) 机器人的感觉运动系统, 用来保持 平衡姿态

弹簧制成的腰椎, 这样就更具仿生特性并且保持平 衡的难度也更大. 如图 15(b)所示, 原人 3 号具有惯性 测量单元, 电机 $M_{\mathrm{L}}$ 和 $M_{\mathrm{R}}$ 分别驱动左轮 $W_{\mathrm{L}}$ 和右轮 $W_{\mathrm{R}}$. 惯性测量单元用来测量机器人的倾角 $\theta$ 以及角速 度 $\mathrm{d} \theta / \mathrm{d} t$.

斯金纳自动机被编程在数字信号处理器中作为 原人 3 号的与姿态和动作相关的感觉运动系统来发 育机器人的平衡技能.

斯金纳自动机的状态向量 $\boldsymbol{s}=(\theta, \dot{\theta})$ 是通过惯性 测量单元测量得到的. 如表 3 , 倾角和倾角速度分别 分成 11 个状态, 因此斯金纳自动机的状态集合中共 有 121 个状态. 这里 $\boldsymbol{s}=\mathbf{0}$ 是原人 3 号的直立姿态. 代 表原人 3 号的趋向性的内能函数定义如下
表 3 倾角和倾角速度的状态分级

\begin{tabular}{ccc}
\hline Level $k$ & $\theta\left(^{\circ}\right)$ & $\mathrm{d} \theta / \mathrm{d} t(\%)$ \\
\hline-5 & $(-\infty,-15]$ & $(-\infty,-60]$ \\
-4 & $(-15,-10]$ & $(-60,-35]$ \\
-3 & $(-10,-6]$ & $(-35,-20]$ \\
-2 & $(-6,-3]$ & $(-20,-10]$ \\
-1 & $(-3,-0.75]$ & $(-10,-2.5]$ \\
0 & $(-0.75,+0.75)$ & $(-2.5,+2.5)$ \\
1 & {$[+0.75,+3)$} & {$[+2.5,+10)$} \\
2 & {$[+3,+6)$} & {$[+10,+20)$} \\
3 & {$[+6,+10)$} & {$[+20,+35)$} \\
4 & {$[+10,+15)$} & {$[+35,+60)$} \\
5 & {$[+15,+\infty)$} & {$[+60,+\infty)$} \\
\hline
\end{tabular}

$$
E^{(S)}(s)=(150 \theta)^{2}+1.8 \times(4500 \theta \dot{\theta})+(30 \dot{\theta})^{2},
$$

其中第 1 项代表了调节角度的趋向性，最后一项代表 了调节角速度的趋向性，中间项是角度和角速度的 耦合.

斯金纳自动机的操作性行为 $\boldsymbol{o}=U_{\mathrm{PWM}}(\in[-3000$, +3000])作用在电机 $M_{\mathrm{L}}$ 和 $M_{\mathrm{R}}$ 上, 作用量是脉宽调制 波. 作用量分为 15 个等级，如表 4 所示，因此斯金纳 自动机的操作性行为集合中有 15 个操作性行为. 被 选择的操作性行为将会作用在电机的伺服系统上来 控制轮子的转速和方向. 这里不考虑操作性行为能 量, $\forall \boldsymbol{o} \in O E^{(O)}(\boldsymbol{o})=0$.

我们用原人 3 号进行了物理实验来展示平衡机 器人如何通过斯金纳自动机用自治的操作性条件反 射的方式学习平衡技能. 斯金纳自动机的初始人工 


\section{表 4 操作性行为分级}

\begin{tabular}{cc}
\hline Level $k$ & $U_{\mathrm{PWM}}$ \\
\hline-7 & -2500 \\
-6 & -1850 \\
-5 & -1250 \\
-4 & -750 \\
-3 & -450 \\
-2 & -250 \\
-1 & -100 \\
0 & 0 \\
1 & +100 \\
2 & +250 \\
3 & +450 \\
4 & +750 \\
5 & +1250 \\
6 & +1850 \\
7 & +2500 \\
\hline
\end{tabular}

温度 $T_{\mathrm{A}}$ 是 $10000^{\circ} \mathrm{C}$, 降温策略如 (16), 系数 $\sigma$ 减小为 0.75 这样物理实验会更快一些. 原人 3 号的底盘上有 保护性支架. 在每个固定温度开始时, 原人 3 号都从 $28^{\circ}$ 倾角启动. 斯金纳自动机的离散时间间隔为 $10 \mathrm{~ms}$. 在每个温度大约需要 $180 \mathrm{~s}$ 来进行操作性学习.

在物理实验过程中, 如果倾角的绝对值大于 $25^{\circ}$, 操作性学习将会停止, 并且比例-微分-积分控制器将 启动来使机器人坚直. 如果倾角的绝对值小于 $5^{\circ}$, 比 例-微分-积分控制器将停止并且机器人开始继续操 作性学习.

机器人进行操作性学习的物理实验结果如图 16 18 所示. 原人 3 号的感觉运动系统以及它的运动 技能发育为了操作性条件反射. 图 17 显示, 当斯金 纳自动机在操作性条件反射过程中降温时, 原人 3 号 逐渐掌握了平衡技能并且最终成为了第一台可以自 主学习平衡技能的两轮机器人. 如图 17 , 每个温度 $T_{A}$ 的内能的均值以及操作性熵都随着斯金纳自动机 的冷却而下降, 这说明了原人 3 号的自组织特性. 我 们录制了视频, 图 18 展示了一些截图.

\section{9 结语}

斯金纳自动机使心理学理论操作性条件反射具 有了计算机程序的形式. 斯金纳自动机从 Metropolis 算法将 Monte Carlo 方法发展为斯金纳算法, 其中操 作性行为(主动,自主的行为)是基本的元素. 如第 3 节

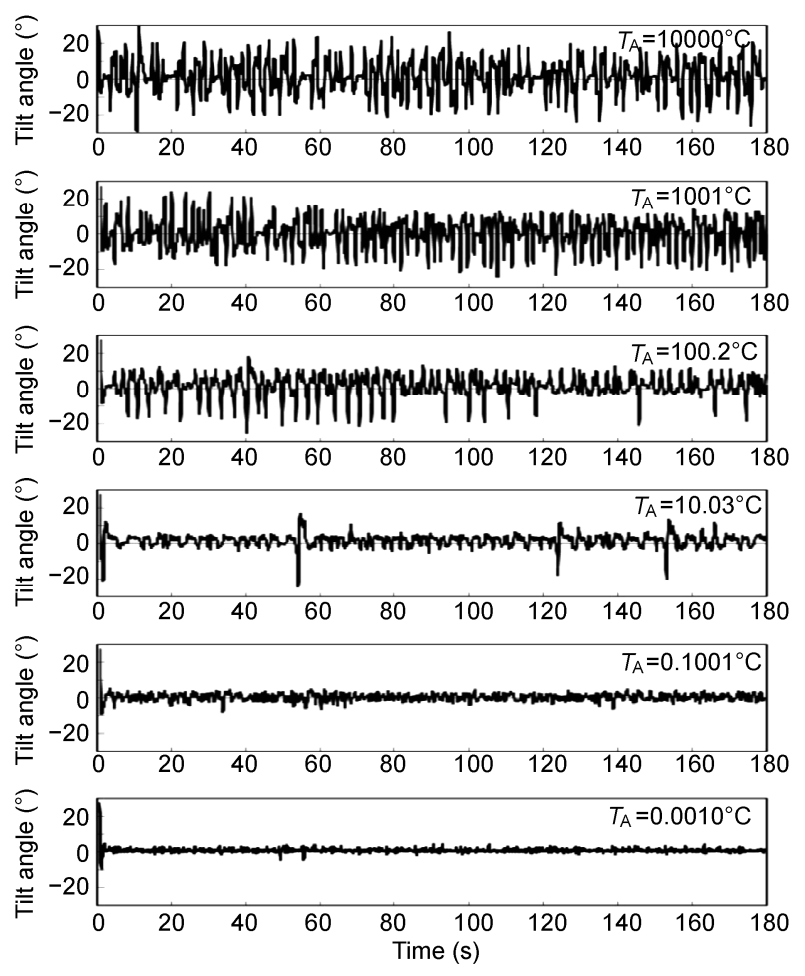

图 16 不同温度 $T_{\mathrm{A}}$ 下的倾角图

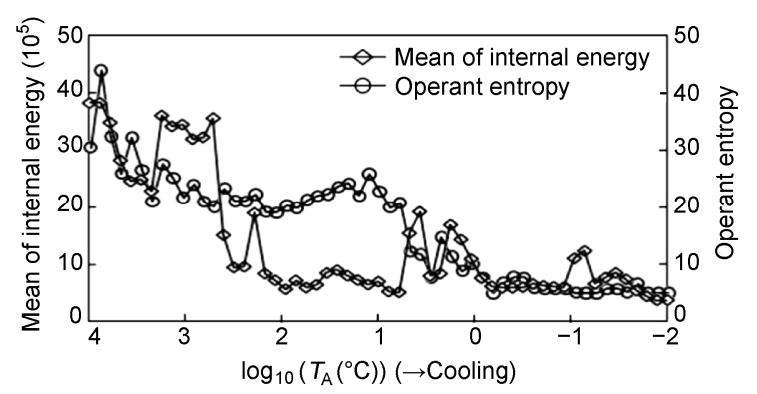

图 17 操作性熵 $\left(T_{\mathrm{A}}\left(=K_{\mathrm{B}} T\right)\right.$ 是人工温度 $)$

所说, 尽管 Monte Carlo 方法可以用来研究操作性行 为 (行为)和结果在反应-刺激条件反射过程中的关系, 但是它既不能选择操作性行为(行为)也不能选择结 果. Metropolis 算法改进了 Monte Carlo 方法, 使它可 以选择 (接受或拒绝)系统的构型或状态. 然而, Metropolis 算法不能直接用在生物系统上. 对于生物, 状态或者构型意味着它行为的结果. 在生物的反应-刺 激条件反射过程中, 一旦结果形成, 是不能被拒绝的. 斯金纳自动机将算法扩展到生物系统上, 另外, 它可 以通过操作性条件反射的方式展现行为选择机制. 斯 金纳自动机通过操作性条件反射选择操作性行为, 并 通过选择导致相应结果的操作性行为来选择结果. 

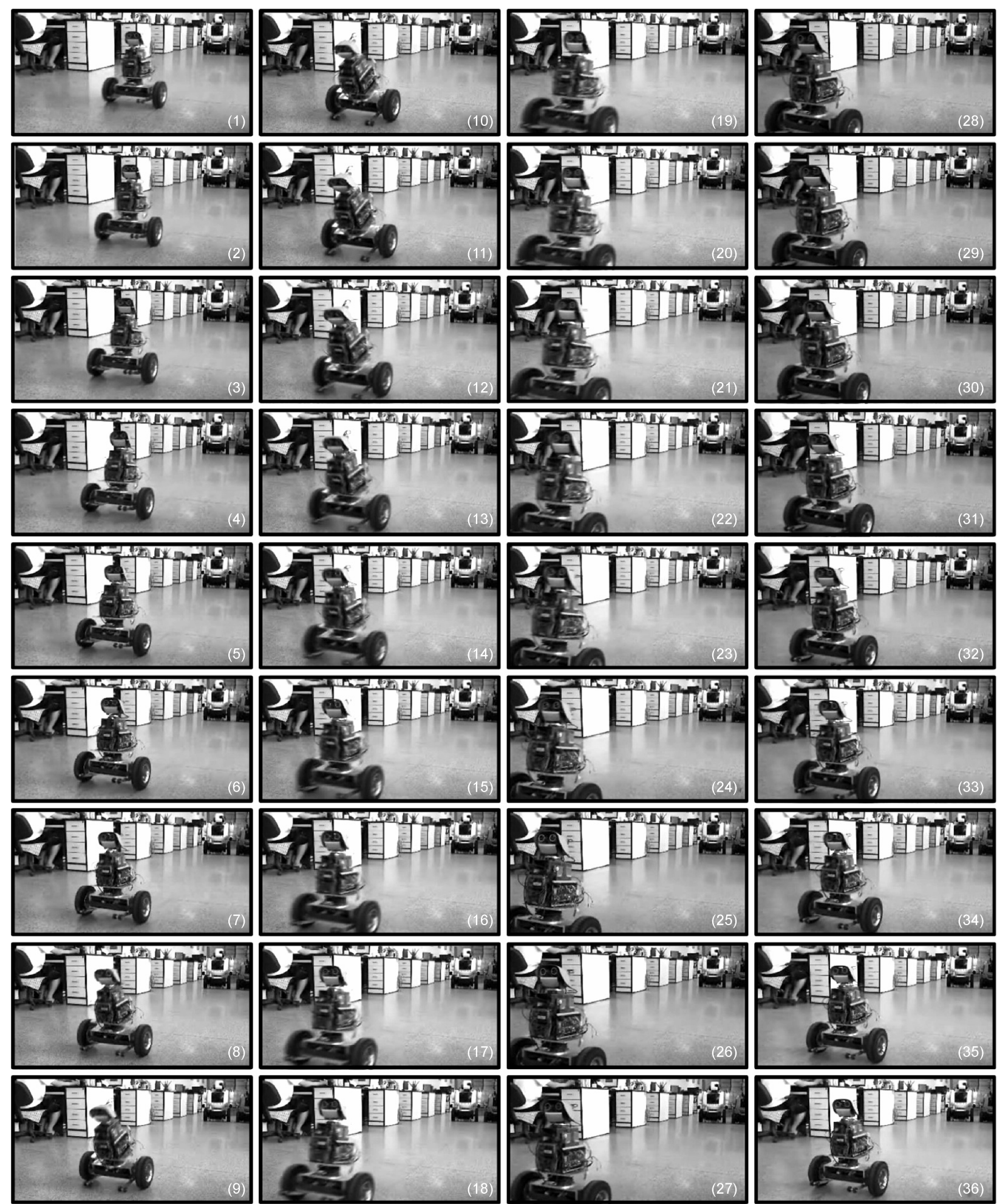

图 18 原人 3 号在摇摆中学习平衡技能 
斯金纳自动机和斯金纳算法的相关工作拓展了 我们对动物认知和学习的认识. 对斯金纳鸽子的研 究为我们通过心理学计算和热力学计算来理解动物 的行为提供了具有意义的例子. 通过维纳虫和原人 3 号的实验展示, 本工作同样对机器人学, 特别是认知 发育机器人具有贡献. 认知发育机器人的目标就是 让人们理解动物的高级认知功能以及机器如何像动 物一样发育地获得认知功能 ${ }^{[47]}$. 认知发育的模型从 感觉运动系统开始, 其中操作性条件反射扮演了重 要的角色. 如同实验中展示的, 斯金纳自动机可以作 为自主智能体的感觉运动系统. 另外, 本工作对于机 器学习, 尤其是自主机器学习有所贡献. 操作性条件 反射是动物学习的基本机制之一. 斯金纳自动机给 人工生命和自主智能体包括移动机器人提供了操作 性条件反射可复制的神经机制, 使它们可以像动物 一样学习. 这样不仅外在, 而且机器可以内在的和动 物一样行动. 作为一名行为心理学家, 斯金纳相信行 为的起因来自于环境而不是内在的心理状态, 如想 法、感觉、感知. 斯金纳宁愿说这些内在心理状态本 身就是行为, 并且像其他行为一样是通过环境的力
量形成和确定的 ${ }^{[48]}$. 当然, 认知和学习包括自学习和 自主学习也都是行为.

生物的行为可以分成两类: 第 1 和 2 级. 第 1 级 是那些我们称作行动的行为, 想法、感觉、感知则属 于第 2 级行为. 第 2 级行为是第 1 级行为的行为, 也是 第 1 级行为的原因. 环境力量形成第 2 级行为, 第 2 级行为又形成第 1 级行为. 我们认为第 2 级行为在自 学习和自主学习中扮演了重要的角色. 它对于自主系 统具有非常的意义, 如人工生命、自主智能体以及移 动机器人, 使它们自主地学习, 成为自主学习者.

斯金纳自动机可以使自主智能体包括机器人成 为自主学习者, 其中操作性行为是第 1 级行为, 代表 特定生物趋向性的能量函数则与第 2 级行为相关. 我 们将斯金纳自动机的操作性条件反射机制称为自主 操作性条件反射.

生物的第 2 级行为和趋向性与内在动机相关 ${ }^{[49}$, ${ }^{50]}$. 在斯金纳自动机中, 与能量函数相关的第 2 级行 为是预先设计好, 并且不可改变的, 不是环境的力量 形成的. 作为第 2 级行为, 斯金纳自动机的内能函数 或者趋向性如何发展将是我们未来的工作.

\section{参考文献}

1 Skinner B F. The Behavior of Organisms. New York: Appleton-Century-Crofts, 1938. 61-116

2 Skinner B F. Science and Human Behavior. New York: Macmillan, 1953. 45-128

3 Thorndike E L. Animal Intelligence: Experimental Studies. Edison: Transaction Publishers, 1911. 241-282

4 Watson J B. Behaviorism. New York: People's Institute, 1924. 141-232

5 Watson J B. Psychology as the behaviorist views it. Psychol Rev, 1913, 20: 158-177

6 Pavlov I P. Conditioned Reflexes: An Investigation of the Physiological Activity of the Cerebral Cortex. London: Oxford University Press, 1927. 219-300

7 Grossberg S. On the dynamics of operant conditioning. J Theor Biol, 1971, 33: 225-255

8 Grossberg S. Classical and instrumental learning by neural networks. In: Rosen R, Snell F, eds. Progress in Theoretical Biology. New York: Academic Press, 1974. 51-141

9 Chang C, Gaudiano P. Application of biological learning theories to mobile robot avoidance and approach behaviors. Advs Complex Syst, 1998, 1: 79-114

10 Touretzky D S, Saksida L M. Operant conditioning in Skinnerbots. Adapt Behav, 1997, 5: 219-247

11 Saksida L M, Raymond S M, Touretzky D S. Shaping robot behavior using principles from instrumental conditioning. Rob Auton Syst, 1997, 22: 231-249

12 Daw N D, Touretzky D S. Operant behavior suggests attentional gating of dopamine system inputs. Neurocomputing, 2001, 38: 1161-1167

13 Itoh K, Miwa H, Matsumoto M, et al. Behavior model of humanoid robots based on operant conditioning. In: Proceedings of the 5th IEEE-RAS International Conference on Humanoid Robots. Tsukuba, 2005. 220-225

14 Itoh K, Onishi Y, Takahashi S, et al. Development of face robot to express various face shapes by moving the parts and outline. In: Proceedings of the 2nd Biennial IEEE/RAS-EMBS International Conference on Biomedical Robotics and Biomechatronics. Scottsdale, 2008. 439-444

15 Sutton R S, Barto A G. Reinforcement Learning: An Introduction. Cambridge, MA: MIT Press, 1998. 1-86 
16 Narendra K S, Thathachar M A L. Learning automata: A survey. IEEE Trans Syst Man Cybern, 1974, SMC-14: 323-334

17 Thathachar M A L, Sastry P S. Varieties of learning automata: An overview. IEEE Trans Syst Man Cybern B Cybern, 2002, 32: 711-722

18 Thathachar M A L, Sastry P S. A new approach to designing reinforcement schemes for learning automata. IEEE Trans Syst Man Cybern, 1985, SMC-15: 168-175

19 Lanctot J K, Oommen B J. Discretized estimator learning automata. IEEE Trans Syst Man Cybern, 1992, 22: 1473-1483

20 Thathachar M A L, Phansalkar V V. Learning the global maximum with parameterized learning automata. IEEE Trans Neural Netw, 1995, 6: 398-406

21 Phansalkar V V, Thathachar M A L. Local and global optimization algorithms for generalized learning automata. Neural Comput, 1995, 7: 950-973

22 Hauwere Y-M De, Vrancx P, Nowé A. Generalized learning automata for multi-agent reinforcement learning. AI Commun, 2010, 23: 311-324

23 Viswanathan R, Narendra K S. A note on the linear reinforcement scheme for variable-structure stochastic automata. IEEE Trans Syst Man Cybern, 1972, SMC-2: 292-294

24 Poznyak S, Najim K. On nonlinear reinforcement schemes. IEEE Trans Automat Contr, 1997, 42: 1002-1004

25 Stoica F, Popa E M. An absolutely expedient learning algorithm for stochastic automata. WSEAS T Comput, 2007, 6: 229-235

26 Stoica F, Popa E M. A new evolutionary reinforcement scheme for stochastic learning automata. In: Mastorakis N E, Mladenov V, Bojkovic Z, et al., eds. The Proceedings of the 12th WSEAS International Conference on Computers, Stevens Point. Wisconsin, 2008. 268-273

27 Simian D, Stoica F. A new nonlinear reinforcement scheme for stochastic learning automata. In: The Proceedings of 12th WSEAS International Conference on Automatic control, Modeling \& Simulation. Catania, 2010. 450-454

28 Metropolis N, Rosenbluth A W, Rosenbluth M N, et al. Equation of state calculations by fast computing machines. J Chem Phys, 1953, 21: $1087-1092$

29 Jorgensen W L. Perspective on 'Equation of state calculations by fast computing machines'. Theor Chem Acc, 2000, 103: 225-227

30 Kirkpatrick S, Gelatt C D, Vecchi M P. Optimization by Simulated Annealing. Science, 1983, 220: 671-680

31 Černý V A. Thermodynamical approach to the travelling salesman problem: an efficient simulation algorithm. J Optim Theory Appl, 1985, 45: $41-51$

32 Horowitz M J. Introduction to Psychodynamics: A New synthesis. New York: Basic Books, 1988. 17-243

33 Palm W J. System Dynamics. 2nd ed. London: McGraw-Hill Science/Engineering/Math, 2009. 172-283

34 Kiese-Himmel C. Verstärkungslernen: Operante Konditionierung. Sprache-Stimme-Gehör, 2010, 34: 1

35 Dayan P, Belleine W. Reward, motivation and reinforcement learning. Neuron, 2002, 36: 285-298

36 Oudeyer P-Y, Kaplan F, Hafner V V. Intrinsic motivation systems for autonomous mental development. IEEE Trans Evolut Comput, 2007, 11: $265-286$

37 Brucke E W, Lectures on Physiology. Vienna: Braumuller, 1874

38 Haynie D. Biological Thermodynamics. Cambridge: Cambridge University Press, 2001. 293-330

39 Nicholls D G, Ferguson S J. Bioenergetics. 4th ed. Europe: Academic Press, 2013. 1-52

40 Hopfield J J. Networks, computations, logic, and noise. In: Proceedings of IEEE First International Conference on Neural Networks. California, 1987. 109-141

41 Neumann J von. Various techniques used in connection with random digits, in Monte Carlo Method. Applied Mathematics Series, vol. 12, Washington D C: U S Department of Commerce, National Bureau of Standards, 1951. 36-38

42 Skinner B F. 'Superstition' in the pigeon. J Exp Psychol, 1948, 38: 168-172

43 Wiener N. Cybernetics: Or Control and Communication in the Animal and the Machine. New York: J Wiley, 1948. 60-132

44 Braitenberg V. Vehicles: Experiments in Synthetic Psychology. USA: The MIT Press, 1986. 95-144

45 Ooi R C. Balancing a two-wheeled autonomous robot. Dissertation of Masteral Degree. Perth: University of Western Australia, 2003. 1-7

46 阮晓钢, 李欣源, 赵伟建, 等. 一种柔性两轮自平衡机器人系统及其运动控制方法. 中国专利, 200910084259.8. 2010-10-9

47 Asada M, Hosoda K, Kuniyoshi Y, et al. Cognitive developmental robotics: A survey. IEEE Trans Auton Ment Dev, 2009, 1: 12-34

48 Wood S E, Wood E G, Boyd D. Mastering the World of Psychology. Boston: Allyn \& Bacon, 2004. 333-354

49 Baranès A, Oudeyer P Y. R-IAC: Robust intrinsically motivated exploration and active learning. IEEE Trans Auton Ment De, 2009, 1: 155-169

50 Oudeyer P Y, Kaplan F. What is intrinsic motivation? A typology of computational approaches. Front Neurorobot, 2007, 1: 1-14 